\title{
AIAA 2004-0612
}

\section{A Structured-Grid Quality Measure for Simulated Hypersonic Flows}

Stephen J. Alter

NASA Langley Research Center

Hampton, Virginia 23681-2199

42nd AIAA Aerospace Sciences Meeting and Exhibit

January 5-8, 2004/Reno, NV

For permission to copy or republish, contact the copyright owner named on the first page. For AIAA-held copyright, write to AIAA Permissions Department, 1801 Alexander Bell Drive, Suite 500, Reston, VA 20191-4344. 


\title{
A Structured-Grid Quality Measure for Simulated Hypersonic Flows
}

\author{
Stephen J. Alter* \\ NASA Langley Research Center Hampton, Virginia 23681-2199
}

\begin{abstract}
A structured-grid quality measure is proposed, combining three traditional measurements: intersection angles, stretching, and curvature. "Quality" assesses whether the grid generated provides the best possible tradeoffs in grid stretching and skewness that enable accurate flow predictions, whereas the grid density is assumed to be a constraint imposed by the available computational resources and the desired resolution of the flow field. The usefulness of this quality measure is assessed by comparing heat transfer predictions from grid convergence studies for grids of varying quality in the range of [0.6-0.8] on an $8^{\circ}$ half angle sphere-cone, at laminar, perfect gas, Mach 10 wind tunnel conditions. All solutions were obtained using the Langley Aerothermodynamic Upwind Relaxation Algorithm with grid adaptation for bow shock alignment and boundary layer resolution. Grid quality for 2D is correlated with surface heat transfer rate accuracy and the convergence capacity of a structured grid for numerical analysis of hypersonic flow fields computed with the Langley Aerothermodynamic Upwind Relaxation Algorithm (LAURA) code. In general, solutions computed on the lower quality grids are less accurate, take longer to converge, and exhibit a lower order of accuracy with respect to grid convergence, although the trends are not monotonic in the proposed grid quality measure.
\end{abstract}

$\begin{array}{ll}\alpha & \text { Angle of attack } \\ \epsilon S & \text { Distance between neighboring points } \\ \epsilon & \begin{array}{l}\text { Stretching factor between neighboring } \\ \text { cells }\end{array} \\ L_{2 \text { norm }} & \text { RSS of residuals in a computation } \\ \theta & \text { Grid line intersection angle } \\ \Theta & \text { Grid line straightness measure } \\ \xi, i & \text { Streamwise computational direction } \\ & \begin{array}{l}\text { and index measured from nose to tail } \\ \text { of body }\end{array} \\ \eta, j & \text { Circumferential computational } \\ & \text { direction and index from top to bottom } \\ \text { of body } & \text { Computational direction and index } \\ \zeta, k & \text { normal to body } \\ q & \text { Heat transfer rate } \\ q_{F R} & \text { Stagnation heating reference from } \\ & \text { Fay and Riddell for a } 1 \text { inch radius sphere } \\ X, Y, Z & \text { Cartesian coordinates }\end{array}$

\section{Introduction}

As the complexity of access-to-space vehicles increases, there is an emphasis for viscous computational fluid dynamics (CFD) to contribute to the determination of aerodynamic and aerothermodynamic characteristics in the preliminary and detailed phases of a design process. ${ }^{1}$ The goal of using these higher fidelity tools is to assess the environment the vehicle encounters during Earth/planetary entry, ${ }^{2,3}$ to reduce aerothermodynamic margins, mitigate risks, and reduce cost prior to construction and flight test-

\footnotetext{
* Senior Aerospace Engineer, Senior Member AIAA

Copyright (C) 2004 by the American Institute of Aeronautics and Astronautics, Inc. No copyright is asserted in the United States under Title 17, U.S. Code. The U.S. Government has a royalty-free license to exercise all rights under the copyright claimed herein for Governmental purposes. All other rights are reserved by the copyright owner.
}

ing. Due to the complexity of hypersonic flow simulations, the grid density required to obtain resolution of flow field gradients is unknown prior to CFD analysis. Reductions in grid density while maintaining grid quality can reduce computation time required to accurately predict flow field phenomenon, thereby making the use of CFD in the design process attractive.

Currently, design tools utilize engineering assumptions and semi-empirical techniques ${ }^{4-7}$ to asses performance characteristics related to aerothermodynamics. These design methods are useful in the determination of vehicle shapes that can be considered for a mission, but they are limited in accuracy, increasing the margins of error that can lead to increased risk or failure of the vehicle to meet mission profiles. Semi-empirical techniques typically ignore viscous effects, which can have a profound effect on a vehicle in hypersonic flows.

Utilizing CFD to accurately predict surface and flow field quantities requires the discretization of a flow domain about the vehicle, such that the best quality of the discretization is obtained. High quality structured grids exhibit near orthogonality and moderate stretching. As it stands, prior to performing a CFD analysis, the quality and resolution of a grid as it relates to accurate flow field predictions is unknown. Grid resolution is dependent on the available computer resources and the sensitivity of flow properties with respect to a grid converged solution. Likewise, trials of different topologies and trade-offs between stretching and skewness may be warranted to determine the most appropriate grid for use with the available computer resources. However, there is no single measure to date for the assessment of grid quality prior to starting a CFD simulation that could determine the applicability of the grid to an accurate prediction.

Current methods used to determine the quality of a grid $^{8-10}$ include the evaluation of parameters such as grid 
line intersection skewness, point-to-point stretching, aspect ratio, cell volume, and Jacobian of the transformation. Typically these measures are individually evaluated to determine if a grid is good enough for use by CFD tools. Single evaluations of these measures are not sufficient to characterize the overall grid quality. Since some of these measures may conflict with one another as they are used to assess grid quality, a combination of the metrics is sought. With respect a structured grid, the combination of the deviation from orthogonality and the degree stretching, in the absence of crossed grid lines, is chosen as the indicator for determining whether or not a CFD solver can accurately and efficiently predict fluid properties on that grid.

This paper establishes a metric which is used to determine the quality of a grid such that a flow field prediction using the Langley Aerothermodynamic Upwind Relaxation Algorithm (LAURA) code, can be obtained with a minimum of point densities. To establish a basis for the effect that structured grid quality and point density has on the accuracy and efficiency of solving the NavierStokes (NS) equations, heat transfer rate predictions are examined for grids of various quality. Heat transfer rate is a basic aerothermodynamic parameter and requires full boundary layer profile resolution in the computational domain to be accurately predicted. The prediction of heat transfer rate is evaluated on a sphere-cone geometry, using the Langley Aerothermodynamic Upwind Relaxation Algorithm $^{11}$ (LAURA) software. Axis-symmetric (2D) and three-dimensional (3D) flow field computations were performed to assess the effects of grid stretching and skewness.

\section{Defining Structured-Grid Quality}

The proposed grid quality measure is abbreviated as GQ. This metric will be used to categorize a grid based on orthogonality, stretching, and straightness, and is given by,

$$
G Q=\frac{\bar{\theta}_{\min } \bar{\Theta}}{\bar{\epsilon}_{\max }}
$$

This measure has been developed based on grid generation experience and commonly used measures. ${ }^{8-10,12}$ It embodies a compromise between a deviation from orthogonality $\left(\bar{\theta}_{\text {min }}\right)$, stretching $\left(\bar{\epsilon}_{\text {max }}\right)$, and straightness $(\bar{\Theta})$ where the best measure is unity $(G Q=1)$. This value is attainable only on a grid that is orthogonal with uniform spacing and straight; a Cartesian grid. Typical values of GQ for grids of interest range from 0.6 to 0.8 .

The minimum of the average planar skewness $\left(\bar{\theta}_{\min }\right)$, which is a measure of how much deviation there is from an orthogonal intersection, is determined as an in-plane quan- tity given by

$$
\begin{aligned}
\left.\theta\right|_{\xi=\text { constant }} & =\frac{2}{\pi} \cos ^{-1}\left(\frac{\vec{r}_{\eta} \cdot \vec{r}_{\zeta}}{\left|\vec{r}_{\eta}\right|\left|\vec{r}_{\zeta}\right|}\right) \\
\left.\theta\right|_{\eta=\text { constant }} & =\frac{2}{\pi} \cos ^{-1}\left(\frac{\vec{r}_{\xi} \cdot \vec{r}_{\zeta}}{\left|\vec{r}_{\xi}\right|\left|\vec{r}_{\zeta}\right|}\right) \\
\left.\theta\right|_{\zeta=\text { constant }} & =\frac{2}{\pi} \cos ^{-1}\left(\frac{\vec{r}_{\xi} \cdot \vec{r}_{\eta}}{\left|\vec{r}_{\xi}\right|\left|\vec{r}_{\eta}\right|}\right)
\end{aligned}
$$

and ranges from 0 as a minimum, for a collapsed cell, to 1 as a maximum, for an orthogonal intersection. Examples of grid quality for orthogonality are shown in figure 1 .

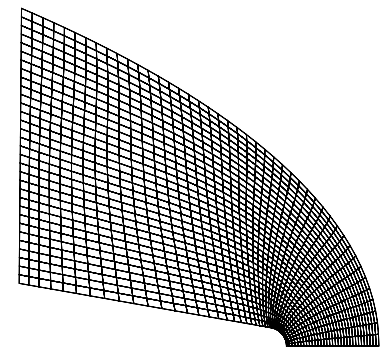

a) Poor orthogonality $(\bar{\theta}=$ $0.6678)$.

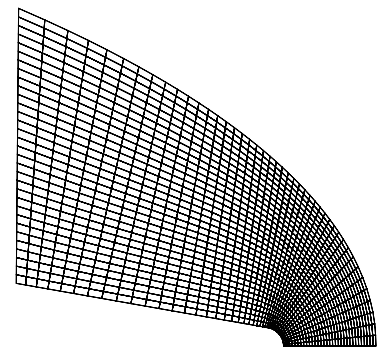

b) Good orthogonality $(\bar{\theta}=$ 0.7814).
Fig. 1 Structured grid quality appearance for orthogonality.

The maximum of the average planar stretching $\left(\bar{\epsilon}_{\max }\right)$, measuring the spacing of one point to the next in a specified computational direction, is given by

$$
\begin{array}{r}
\epsilon_{\xi}=\frac{\max \left(\left|\vec{r}_{\xi}\right|^{+},\left|\vec{r}_{\xi}\right|^{-}\right)}{\min \left(\left|\vec{r}_{\xi}\right|^{+},\left|\vec{r}_{\xi}\right|^{-}\right)} \\
\epsilon_{\eta}=\frac{\max \left(\left|\vec{r}_{\eta}\right|^{+},\left|\vec{r}_{\eta}\right|^{-}\right)}{\min \left(\left|\vec{r}_{\eta}\right|^{+},\left|\vec{r}_{\eta}\right|^{-}\right)} \\
\epsilon_{\zeta}=\frac{\max \left(\left|\vec{r}_{\zeta}\right|^{+},\left|\vec{r}_{\zeta}\right|^{-}\right)}{\min \left(\left|\vec{r}_{\zeta}\right|^{+},\left|\vec{r}_{\zeta}\right|^{-}\right)}
\end{array}
$$

and ranges from 1 as a minimum, for uniform spacing, to infinity. Poor grid stretching is identified by abrupt variations in the spacing of grid points. Examples of good and poor stretching are illustrated in figure 2.

Finally, grid line straightness $(\bar{\Theta})$, which measures the extra distance traversed between the wall and the outer boundary, is computed using

$$
\Theta=\frac{\left.S\right|_{\left(\zeta_{\min } \rightarrow \zeta_{\max }\right)}}{\sum_{\zeta=1}^{\zeta_{\max }}|\vec{r}|_{\zeta}}
$$

Unlike stretching and orthogonality, straightness is only measured in the body-normal direction because it is useful as a grid adaption parameter. The grid line straightness measure ranges from 0 as a minimum, for a grid line that 


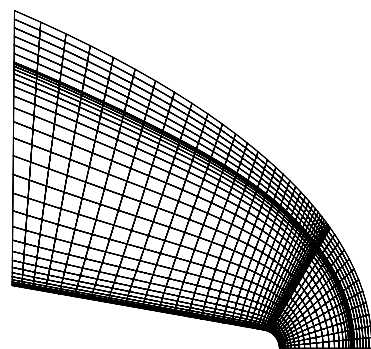

a) Poor stretching $\left(\overline{\epsilon_{\xi}}=1.18, \overline{\epsilon_{\zeta}}=1.31\right)$.

Fig. 2 Structured grid quality appearance for stretching in capturing flow features.

begins and ends at the same point, to 1 as a maximum value for a straight line. If a flow solver is not sensitive to curvature in the body-normal direction, the straightness measure can be disabled by setting it equal to one. The grids in figure 3 illustrate poor and good straightness.

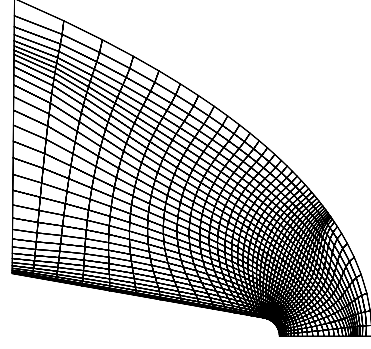

a) Poor straightness $(\bar{\Theta}=$ $0.9577)$.

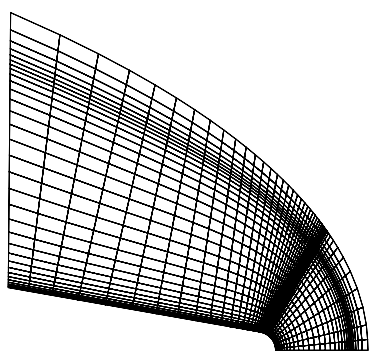

b) Good straightness $(\bar{\Theta}=$ 1.0000).
Fig. 3 Structured grid quality appearance for straightness.

While the GQ is a single value as defined in terms of the three components including, orthogonality, stretching, and straightness, evaluations of the the usefulness of the measure will be performed by primarily degrading one component at a time. Since these measures are coupled to one another, trade-offs between stretching and orthogonality are made to determine the effects each has on the measure and the accuracy or consistency of flow field simulations.

\section{Geometry and Grids}

In order to assess the capacity of a grid to accurately predict a flow field solutions are generated for an eight inch, $8^{\circ}$ half angle sphere-cone with a half inch nose radius.

\section{D Grid Generation}

Two dimensional surface grids for the sphere-cone shape are constructed using a system of elliptic partial differential equations (PDEs). Use of such a tool is more akin to modern practices for grid generation in CFD, instead of developing a contrived structured grid. Initially, a high quality grid is constructed such that grid resolution is provided at the sphere-cone juncture. Increasing grid resolution in this region allows for flow field solutions computed on lower quality grids to be affected downstream from this geometric juncture. Orthogonal grid lines emanating from the wall are constructed and continued through the domain until they reach the initial outer boundary, as shown in figure 4 . The initial high quality grid has a GQ of 0.794 .

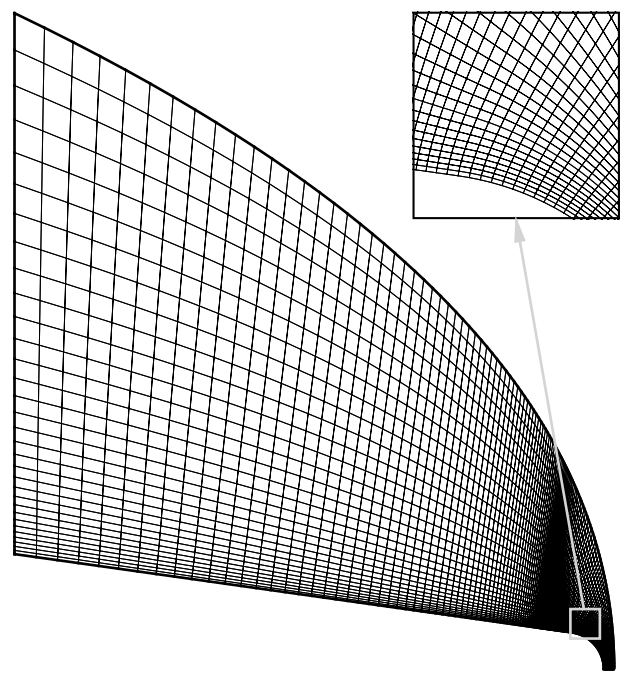

Fig. 4 High quality structured grid (GQ=.794).

Construction of the lower quality meshes begins by retaining the edges and point distributions of the high quality mesh, and regenerating the interior grid with twodimensional trans-finite interpolation ${ }^{13}$ (2DTF). This grid regeneration method isolates the effect of orthogonality relative to stretching to determine incremental effects of quality degradation. This results in the slightly skewed mesh shown in figure 5. A significantly skewed mesh is generated by using the elliptic PDEs to reduce the control used in development of of the high quality mesh, and produces a grid illustrated in figure 6. The GQ measures drop from 0.763 to 0.709 for these grids, which reflects the orthogonality degradation or increased skewness. The slightly skewed mesh is not adversely affected by grid line curvature but the highly skewed mesh has a significant degradation in grid line straightness.

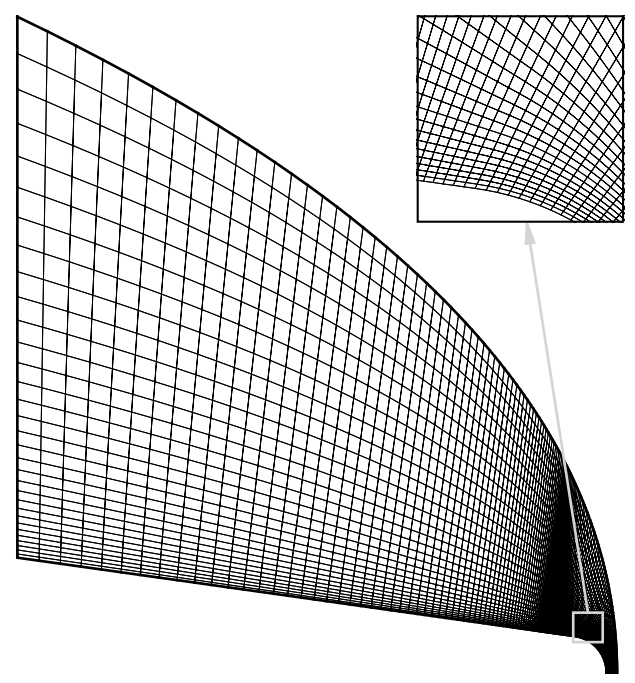

Fig. 5 Slightly skewed (GQ=.763) structured grid. 


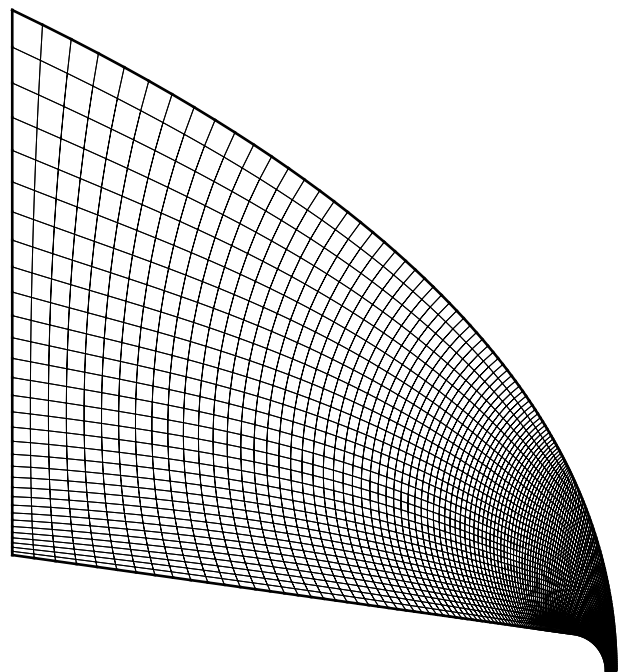

Fig. 6 Highly skewed (GQ=.709) structured grid.

In order to isolate grid stretching effects from grid skewness, the high quality mesh is modified with the volume grid manipulator $^{14}$ (VGM) using parametric re-mapping ${ }^{15}$ (PRM). This technique allows the growth and reduction of cell sizes in the streamwise direction of the high quality mesh. Every eighth cell in the high quality mesh is increased by $25 \%$ by adding a point from the first cell to the eighth cell. This pattern is repeated for every eight cell interval along the streamwise direction, for the length of the sphere-cone geometry, and produces a grid shown in figure 7.

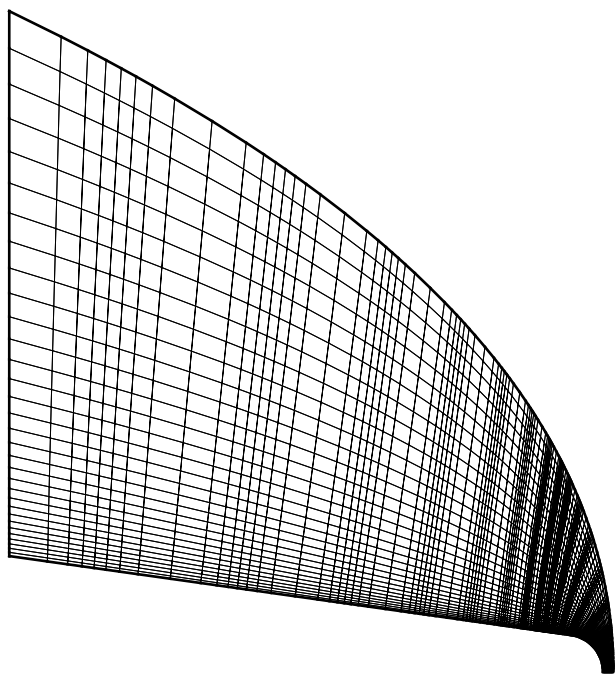

Fig. 7 Highly stretched (GQ=.614) structured grid.

Stretching in the region from the body to outer boundary is not evaluated because grid adaptation in this direction is used to capture the boundary layer and outer bow shock of the flow domain. Since the stretched grid is based on the high quality grid, the interior has a high degree of orthogonality, is orthogonal to the wall, and has straight lines from the wall to the outer boundary. Only the grid distribution function has been altered, enabling the isolation of grid stretching from grid skewness.

\section{D Grid Generation}

Volume grid generation is more difficult with respect to controlling grid quality, but is performed using a similar approach to the 2D surface methods. Since an angle of attack will be simulated, the $2 \mathrm{D}$ high quality mesh is expanded for the leeside symmetry plane and contracted for the wind side symmetry plane. The volume is completed by adding a circular exit plane and transitioning the leeside symmetry plane to the windside, producing a singularity on the sphere. Since singular grids can produce spurious results with finite volume schemes, the nose region is reconstructed by rebuilding the line that extends from the singularity to the thirty-third point downstream. Singularity free grids are built on the surface of the nose, and the faces to the volume grid are complete. The volume is initialized with 3DTFI and subsequently smoothed with the 3DGRAPE/AL ${ }^{16}$ (Three-Dimensional Multiblock Grids about Anything by Poisson's Equations with Ames and Langley upgrades) using anisotropic Lagrange based trans-finite interpolation to provide orthogonality at all faces except the outer boundary. The outer boundary source terms are formulated to enable straight grid lines to traverse from the wall to the outer boundary. The result is a high quality volume grid, shown with the wall grid in figure 8 . Due to the single flow boundary condition per face requirement of the LAURA software, the grid is decomposed at the nose interface to the cone. Generation of the blocks was performed to place an equal number of grid points in each block, resulting in a load balanced grid for cluster computing with 14 grid blocks, as illustrated by the red block boundary lines of the topology about the spherecone.

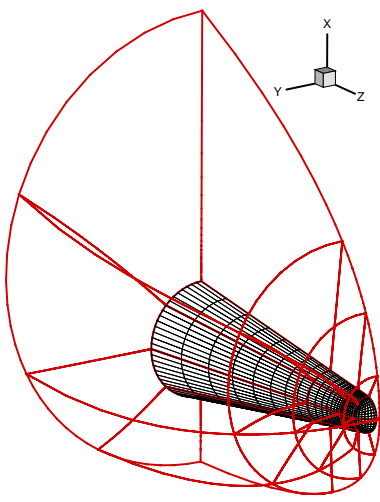

a) Full domain.

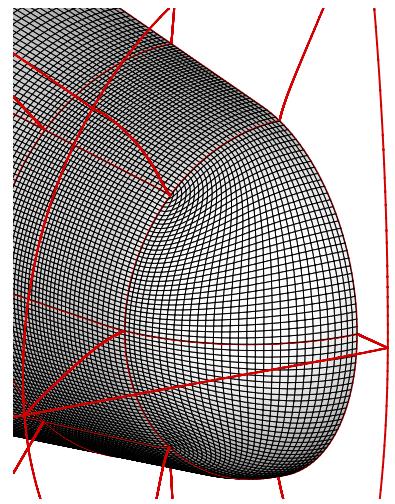

b) Nose region.
Fig. 8 High quality volume grid and wall for 3D CFD simulations.

To evaluate the effects of stretching and grid skewness as a comparison to the high quality grid in $3 \mathrm{D}$, three more meshes were generated, using the high quality mesh as the initial grid definition. Since a contrived solution was not sought, the high quality grid was modified using 2DTFI and 3DTFI, with grid distribution function changes on edges to degrade grid skewness. By using the high quality mesh as a basis for the lower quality grid construction, the wall grid on the cone is maintained for differencing the solutions. 
A slightly skewed grid was generated by re-initializing the nose and body of the high quality grid with 2DTFI and projecting the grids to the geometry. Symmetry and exit planes were generated with 2DTFI, and the volume grid initialized with 3DTFI. To make an even more skewed mesh, the normalized grid distribution functions were copied from the wall to the outer boundary. The same grid generation process used for the slightly skewed 3D mesh was used, and a significantly skewed volume mesh resulted. The 2DTFI produced some grid line skewness at the boundaries of the flow domain, which were propagated onto the interior with 3DTFI. Likewise, the stretched grid was generated by reducing the cell size of the eighth cell by $25 \%$ in the streamwise and cross-sectional directions, and redistributing the remaining seven cells to have a gradual change within each eighth cell interval. By comparison to the high quality mesh, the skewed, highly skewed, and stretched meshes are illustrated with representative crosssectional and streamwise planes shown in figures 9 and 10 , respectively.

Close inspection of the representative surface grids in figures 9 and 10 indicate that the skewed meshes have minimal stretching, while the stretched grid has minimal skewness. Hence, the grid generation procedures have produced a set of volume grids that nearly isolate the effects of skewness and stretching from the baseline high quality mesh. This will enable characterization of cause and effect when assessing the flow solutions computed on these volume grids.

\section{Flow Solver and Method}

\section{Grid Quality Impact}

Assessment of grid quality with respect to flow solution accuracy is performed by comparing CFD and experimental data for the sphere-cone which matches a previously conducted wind tunnel test using the phosphor thermography technique. ${ }^{17}$ Grid quality is measured with orthogonality, stretching, and straightness, but not grid resolution. Grid resolution is only considered when determining the density of a grid that produces a solution that is independent of the grid; a condition of grid convergence. The high quality grid that has the required density to obtain a grid independent solution is considered a high fidelity grid. Fidelity of a grid in this definition is a combination of both quality and grid resolution.

The experimental heat transfer rates for the wind tunnel data have a reported uncertainty of $10-15 \%$. A zero degree angle of attack is used for the 2D simulations while 3D simulations were performed at at angle of attack of 15 degrees. The flow simulations use laminar perfect gas wind tunnel conditions at a Mach number of 10, a Reynold's number of $3.7533 \times 10^{5} /$ foot, a constant wall temperature of $300 \mathrm{~K}$, and a density of $4.455 \times 10^{-3} \mathrm{~kg} / \mathrm{m}^{3}$. Both $2 \mathrm{D}$ and 3D simulations were performed with the same wind tunnel conditions on an $8^{\circ}$ half angle sperically blunted cone with a nose radius equal to a half an inch.

The flow solver used for the CFD simulations in this

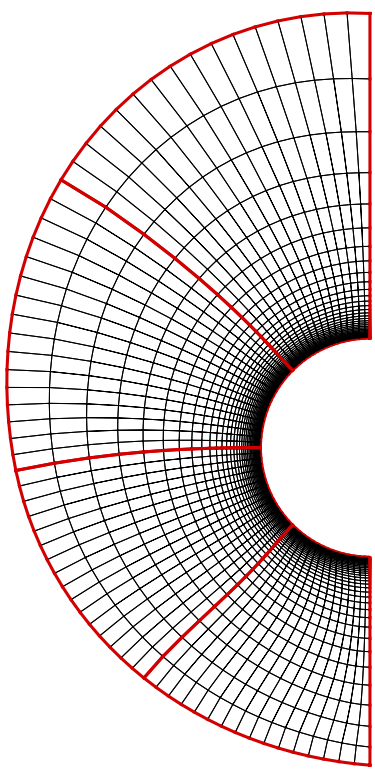

a) High quality.

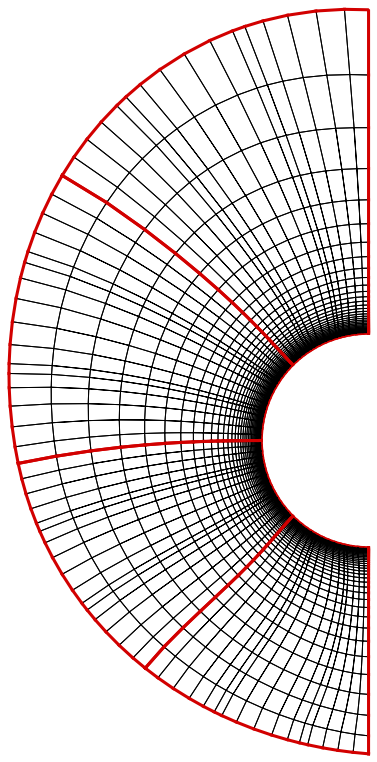

c) Stretched.

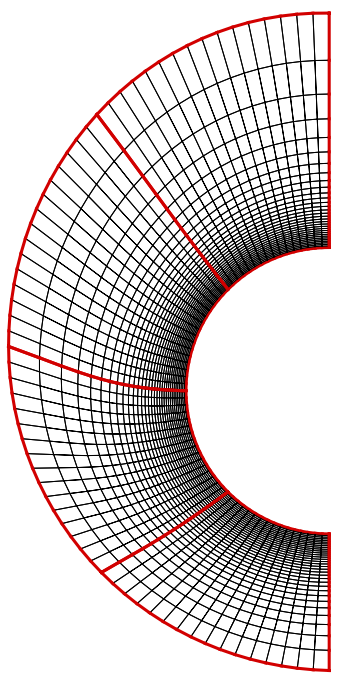

b) Moderately skewed.

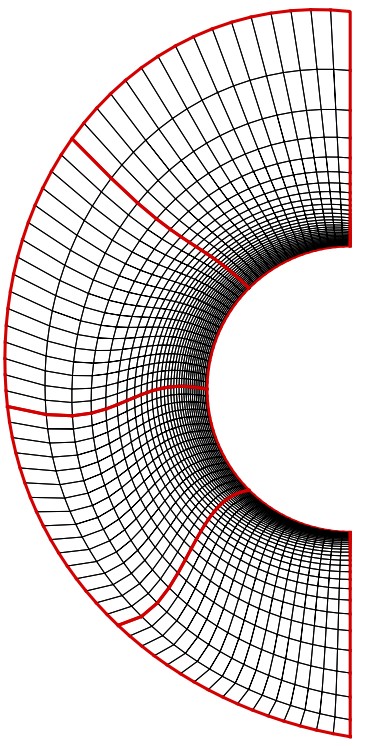

d) Highly skewed.
Fig. 9 Comparison of representative 3D cross-sectional for four different quality meshes, coarsened for visibility.

paper is the LAURA ${ }^{11}$ code, a second-order accurate flux difference splitting finite volume scheme. This code solves the TLNS and NS equations, where the inviscid flux is constructed using Roe's flux difference splitting ${ }^{18}$ and Harten's entropy fix, ${ }^{19}$ with second order corrections based on Yee's symmetric total variation diminishing (TVD) scheme. ${ }^{20}$ The solver makes use of a grid adaptation procedure called ALGNSHK (align-shock) that aligns the volume grid to the bow shock and clusters the grid to the boundary layer. This technique provides a powerful mechanism to increase the efficiency of the available grid points to be used in the simulation of the flow field, at the expense of introducing stretching and being less robust for curved body-to-shock 


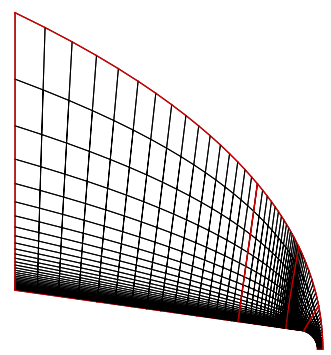

a) High quality.

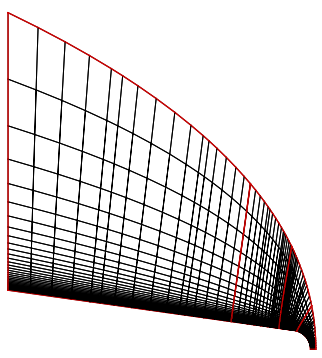

c) Stretched.

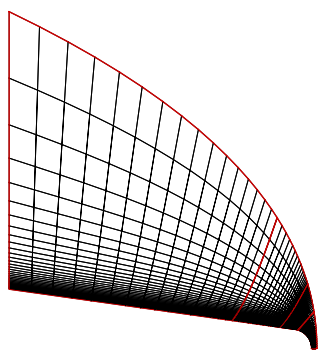

b) Moderately skewed.

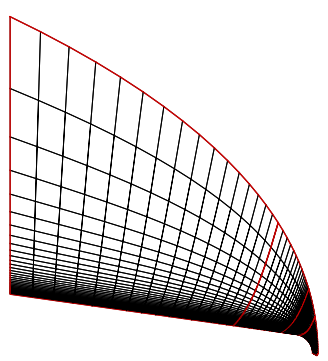

d) Highly skewed.
Fig. 10 Comparison of representative 3D streamwise planes for four different quality meshes, coarsened for visibility.

direction grid lines.

\section{Solution Strategy}

LAURA solutions were converged 10 orders of magnitude for each reported 2D solution. For 3D simulations, a convergence criteria of integrated maximum heat transfer rate change less than $0.1 \%$ with 2000 iterations between consecutive solutions was used. Different transient paths to steady state convergence for both $2 \mathrm{D}$ and $3 \mathrm{D}$ were tried to verify process independence. Grid convergence rates are measured by the maximum point change in surface heat transfer for each doubling of the grid size. Grid convergence comparisons are performed by differencing a fine mesh, which has twice as many points in all computational directions, with a coarser mesh, and normalizing the results by the finer mesh solution.

\section{Heat Transfer Rate Grid Convergence in 2D}

The converged flow field results for the different grid densities using the axis-symmetric high quality grid are shown in figure 11. Computations with various grid densities are compared to experimental data, whereby predicted and measured laminar heat transfer rate $(q)$ are nondimensionalized by the Fay and Riddell ${ }^{21}$ heat transfer rate of $13.60 \mathrm{~W} / \mathrm{cm}^{2}$ for a one inch radius sphere. ${ }^{17}$ It should be noted that the Fay and Riddell heating on a $\frac{1}{2}$ " radius sphere is 1.414 times the heating on a 1" radius sphere. The heating presented in figure 11 is plotted along the surface of the geometry, where $\xi / \xi_{\max }$ represents the percentage from the nose to the base.

Although the agreement between experimentally obtained and CFD predicted heating appears to be significant along the cone, the measurement uncertainty of the experimental data in this region is closer to $15 \%$. However, agree-

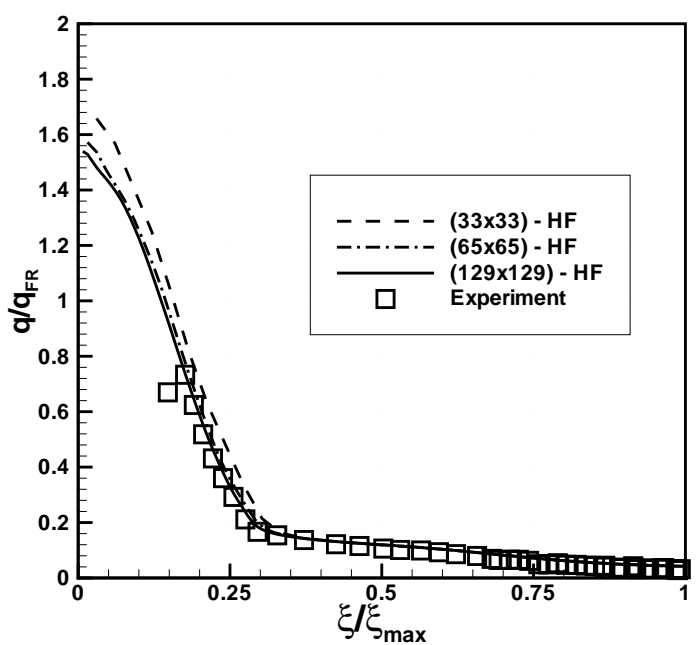

Fig. 11 Comparison of TLNS computed and experimental data for the high quality grid with a GQ of 0.794 .

ment of the experimental data and CFD prediction near the sphere-cone junction is better than the cone because the heating is more significant, due to reduced measurement uncertainty in the experimental data. In this region, the comparison of the different grid densities shows that as the grid density increases the agreement of the computation increases. As mentioned earlier, fidelity is a combination of grid resolution and quality. Since the solution on the grid with a point density of $(65 \times 65)$ accurately predicts the heating, this grid is considered to be a high fidelity grid. Checking for grid convergence, the solutions from the fine to previously coarse meshes are compared, and shown in figure 12. For this paper, a 2D heating solution is considered to be grid converged if the change in heating from one grid density to the next is less than or equal to $3 \%$, the maximum difference in heating between medium and fine high quality meshes.

For the slightly skewed grid, figure 5, the grid convergence is shown in figure 13. The grid convergence indicates that the change in heating from the $(65 \times 65)$ grid density to $(129 \times 129)$ grid density is $2.9 \%$. Although the medium mesh satisfies the grid convergence criteria, there is a measurable increase in the solution change with grid refinement, $2.9 \%$ versus $2.7 \%$, as compared to the high quality. This slightly skewed grid is visually very similar to the high fidelity grid, but the grid quality measure is able to identify a reduction in quality.

For the highly skewed grid, figure 6, the grid convergence is shown in figure 14 . The grid convergence indicates a maximum point heating change of approximately $1.7 \%$ from the $(65 \times 65)$ to the $(129 \times 129)$ grid resolution. In this case, the difference in heating is less than the slightly skewed mesh, yet the differences between the coarse and medium grids are significantly greater than the slightly skewed mesh. Since the grid is significantly different in 


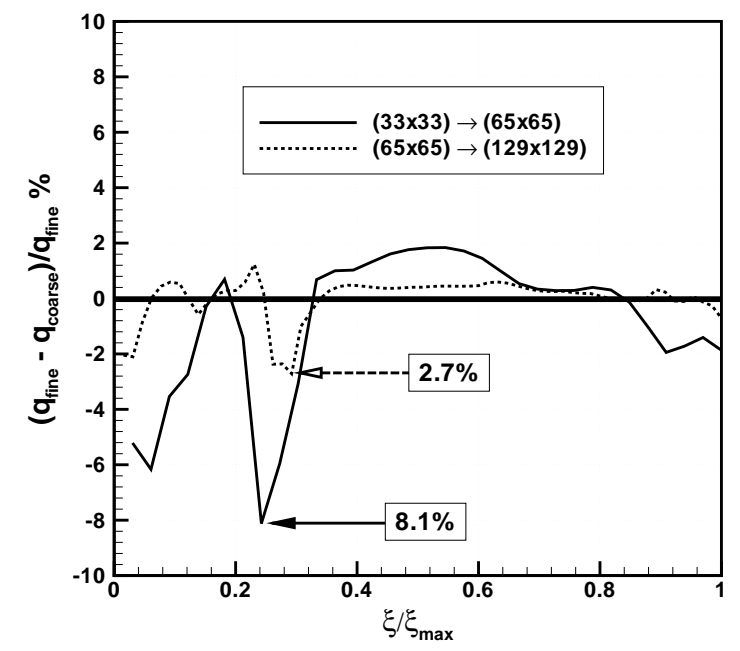

Fig. 12 Grid convergence of the high quality grid (GQ=.794).

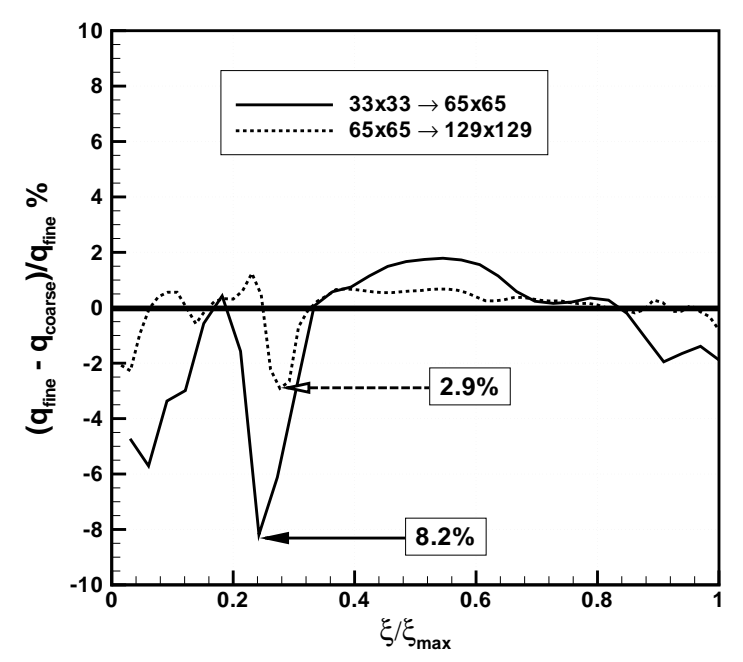

Fig. 13 Grid convergence of the slightly skewed grid case (GQ=.763).

point distribution at the outer boundary, and interior curvature, comparisons between the skewed meshes may not be valid. Rather, consistency between the highly skewed mesh and the high quality mesh is more likely the better indicator of solution accuracy.

For the highly stretched grid of figure 7, with a $\mathrm{GQ}=.614$, grid convergence shown in figure 15 is not achieved for the $(33 \times 33)$ grid density, nor the $(65 \times 65)$ point density. No further solutions were attempted because there was no indication that grid convergence would occur. Under the conditions of convergence, and based on the quality measure, the CFD results from the significantly stretched grid indicates that a stretching of 1.75 is too excessive to obtain accurate analyses.

By comparing the grid converged solutions for the

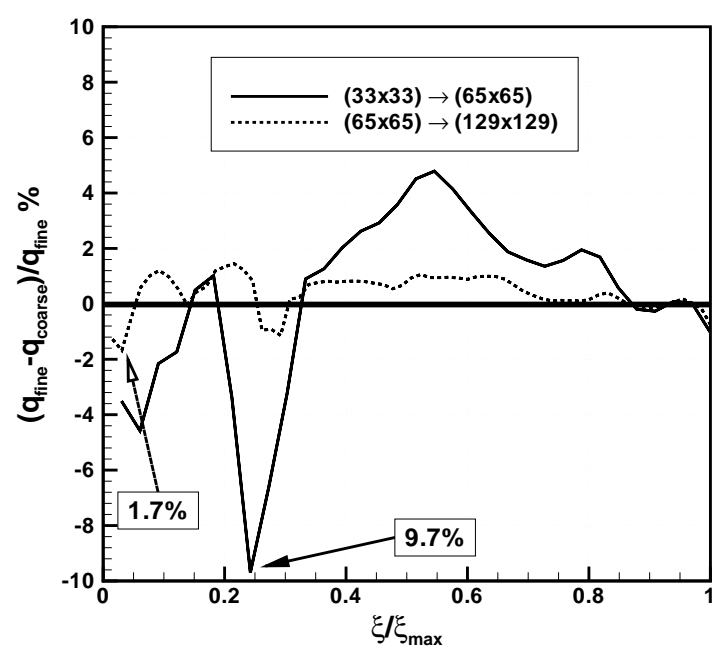

Fig. 14 Grid convergence of the highly skewed grid case (GQ=.709).

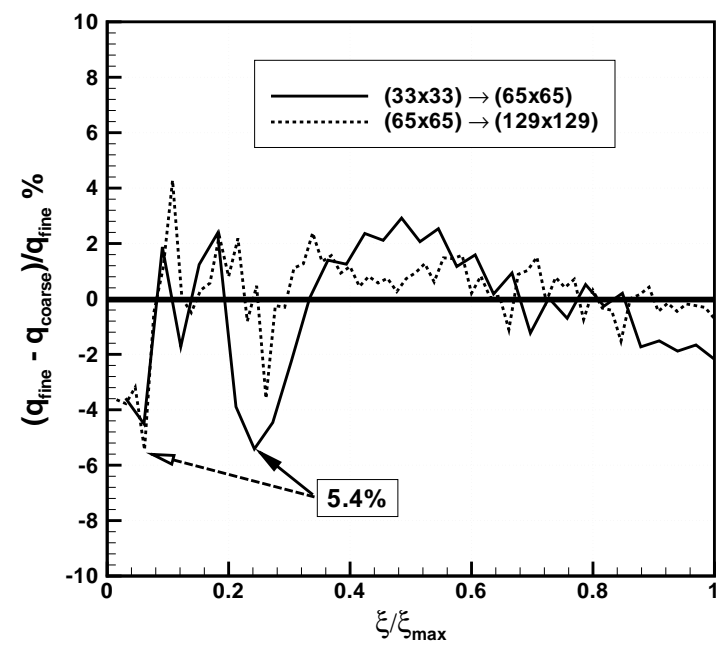

Fig. 15 Lack of grid convergence for the highly stretched grid case $(G Q=.614)$.

high fidelity, slightly skewed, highly skewed, and highly stretched grids, as shown in figure 16, the differences in heating indicate inconsistencies between the solutions. The comparison of heating for the skewed to high fidelity grids indicate solution differences that are measurable but within the grid convergence tolerance. However, the highly skewed mesh identifies significant degradation of accuracy by as much as $4.7 \%$, which is larger than the grid convergence error criteria. Thus, as the skewness of the grid is increased, the consistency of the solution with respect to the high quality mesh decreases. Clearly the stretched grid is significantly different from the high fidelity and is outside of the grid convergence tolerance. Hence, the same degree of grid convergence is not obtained on the stretched grid. 


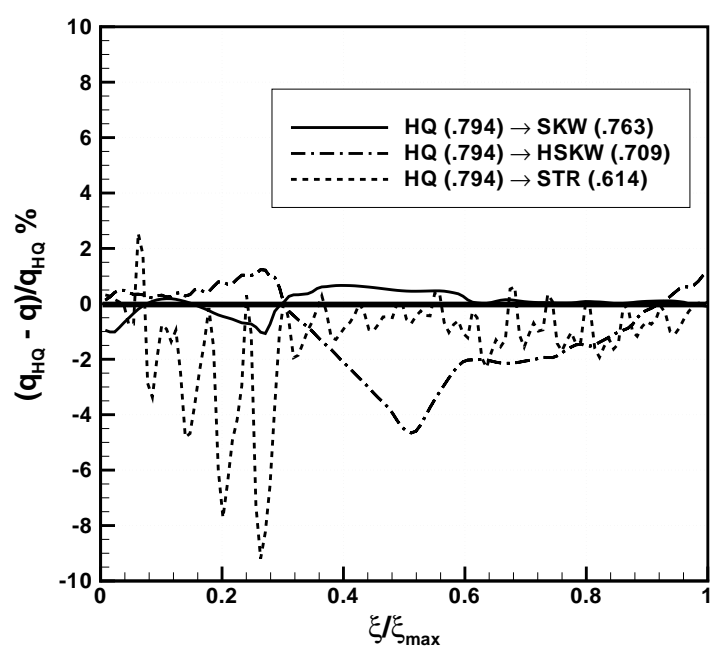

Fig. 16 Comparison of heating between the high fidelity, skewed, and stretched grid converged solutions.

A correlation of the GQ metric is developed by comparing the surface heat transfer agreement between the high quality, skewed, and stretched grids where the largest magnitude of variation from heating to the high quality mesh is considered. As shown in figure 17, as the GQ measure decreases so does the solution consistency and accuracy. Thus the measure does have some merit in assessing the viability of predicting the capacity of a grid to be useful in obtaining accurate heating.

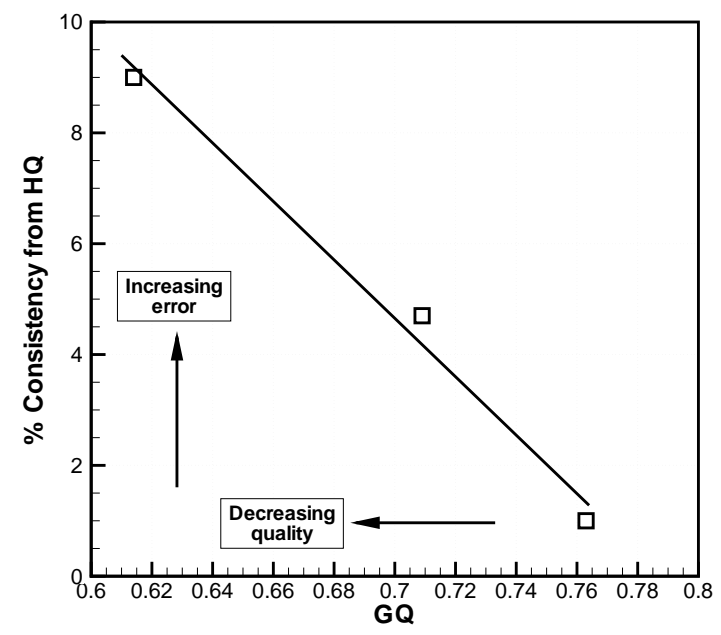

Fig. 17 Grid Quality metric correlation with axis-symmetric flow simulation accuracy.

\section{Heat Transfer Rate Grid Convergence in 3D}

For the 3D assessment, a high quality volume grid, two different skewed volume grids, and a stretched volume grid were developed. As noted in the grid generation section of this paper, the solution of elliptic PDEs was used only in the construction of the highest quality volume grid, and not for the lower quality 3D grids. Instead, algebraic methods were used to generate the skewed and stretched meshes.

The CFD simulations performed with the 3D volume grids were similar to the $2 \mathrm{D}$ simulations, but performed at an angle of attack of 15 degrees. A different convergence criteria was used for the $3 \mathrm{D}$ computations by comparison to the $2 \mathrm{D}$ predictions because machine zero was not approachable with the 3D grids due to time required to reach that level of convergence. A simulation was considered converged when the $L_{2 n o r m}$ approached zero, less than $1.0 \mathrm{E}-6$ and the surface heat transfer rate changed less than $0.1 \%$ between 2000 iterations. CFD simulations were performed by solving a coarse mesh with every fourth point in the original volume grid, constituting 14 blocks of $(9 \times 9 \times 32)$, a medium mesh with a grid density of $(17 \times 17 \times 64)$, and the original fine mesh density of $(33 \times 33 \times 129)$. The coarse grid was adapted with a cell Reynold's number of 4 , and no adaptation was performed on the medium or fine grids. Only grid doubling was used to refine the adapted volume grids. Using two successive grid doubling procedures, the projected cell Reynold's number at the wall was 1.0. This process ensures the correct usage of the Richardson Extrapolation method for error assessment. ${ }^{22}$ The maximum stretching that results from this procedure is significantly less than 1.2 , which is well within the tolerable limit for LAURA.

To improve the accuracy of viscous gradients modeling, and the leeside separation shown in figure 18, thin-layer viscous terms were retained in the cross-flow and bodynormal directions. Also illustrated in figure 18 is the block boundary coinciding with the separation region. Though an unfortunate selection of the block boundary location, the block boundary was not specifically placed in the separation region. Rather, the block boundary location was a result of equal spacing of grid points circumferentially around the cone and a decomposition required to obtain equally dimensioned blocks.

Since the accuracy of the flow field variables has not been validated with experimental data, the consistency among the different volume grids is evaluated. The high quality grid solutions are used as the baseline for comparisons. Differences in surface heat transfer were computed with respect to the high quality mesh, and are shown in figure 19 for the three low quality grids, and tabulated in table 1 .

\begin{tabular}{ccc}
$\begin{array}{c}\text { Grids } \\
\text { Differenced from } \\
\text { Quality mesh }\end{array}$ & $\begin{array}{c}\text { Maximum } \\
\text { Pressure } \\
\text { (\% diff.) }\end{array}$ & $\begin{array}{c}\text { Maximum } \\
\text { Heating } \\
\text { (\% diff.) }\end{array}$ \\
\hline Stretched & 2.67 & 4.85 \\
Moderately skewed & 5.83 & 24.7 \\
Highly skewed & 5.10 & 20.9 \\
\hline
\end{tabular}

Table 1 Differences in heating and pressure between a quality mesh and poorly generated meshes. 


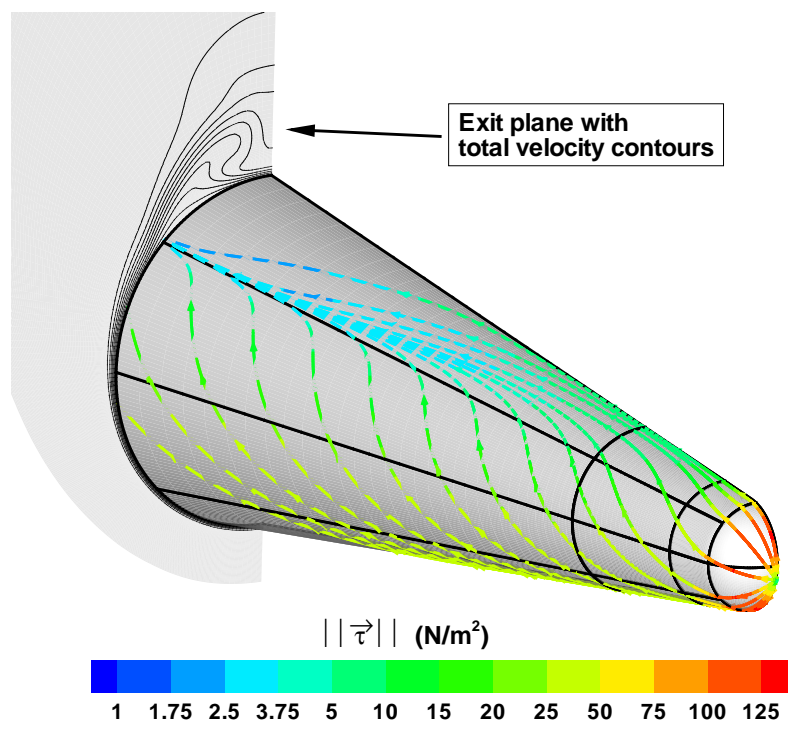

Fig. 18 Leeside separation region on the high quality grid.

Counter to the axis-symmetric results, the heating differences between the quality and stretched meshes shown in figure 19a is small, but measurable. The peak heating difference occurs outside of the separation region, and is focused on the side of the vehicle near the nose. Illustrated in figure $20 \mathrm{a}$, the peak pressure differences occur in the same region.

Results for skewed meshes shown in figures 19b,c and $20 \mathrm{~b}, \mathrm{c}$ indicate similar trends, but the differences are much more significant. Although the surface heating and pressure percent differences are largest in the separated region where the magnitudes of both quantities are small, there are noticeable differences in the nose region that should not be ignored. In the nose region, the heating differences for the quality and skewed meshes illustrate that increasing skewness reduces the consistency of the solution to the higher quality grid, as shown in table 2 .

\begin{tabular}{ccc}
$\begin{array}{c}\text { Different } \\
\text { Grid } \\
\text { Quality }\end{array}$ & $\begin{array}{c}\text { Minimum } \\
\text { Average } \\
\text { Skewness }\end{array}$ & $\begin{array}{c}\text { Maximum } \\
\text { Heating } \\
\text { (\% diff.) }\end{array}$ \\
\hline High Quality & 0.7718 & - \\
Moderately skewed & 0.7298 & 24.7 \\
Highly skewed & 0.7266 & 20.9 \\
\hline
\end{tabular}

Table 2 Global skewness effects on heating.

The highly skewed mesh has skewness at approximately $90 \%$ from orthogonality just past the sphere-cone interface. In the heating and pressure, this region is characterized by measurable differences to the quality mesh. The problem in this region, as well as the separation region, stems from the missing cross-derivative terms in the TLNS equations; portions of the FNS equations that are assumed to be insignificant. The difference between TLNS and FNS equations is the neglecting of the cross-derivative viscous terms. Due to the heating differences, clearly retaining these terms in the presence of skewness is important to accurately capture

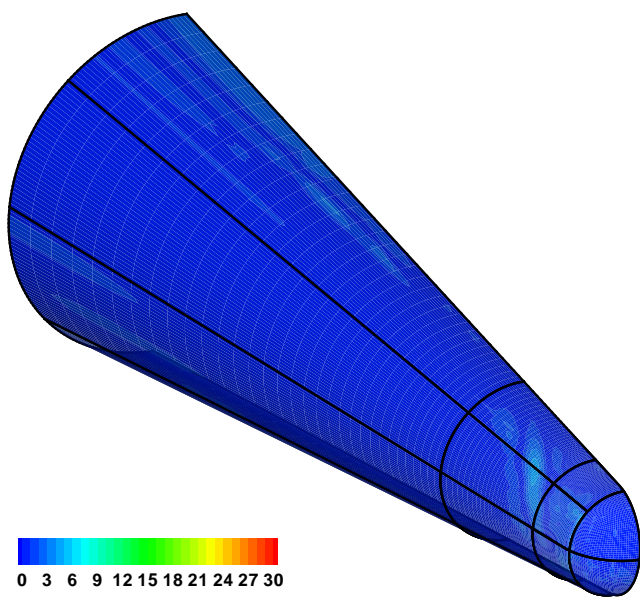

a) Stretched.

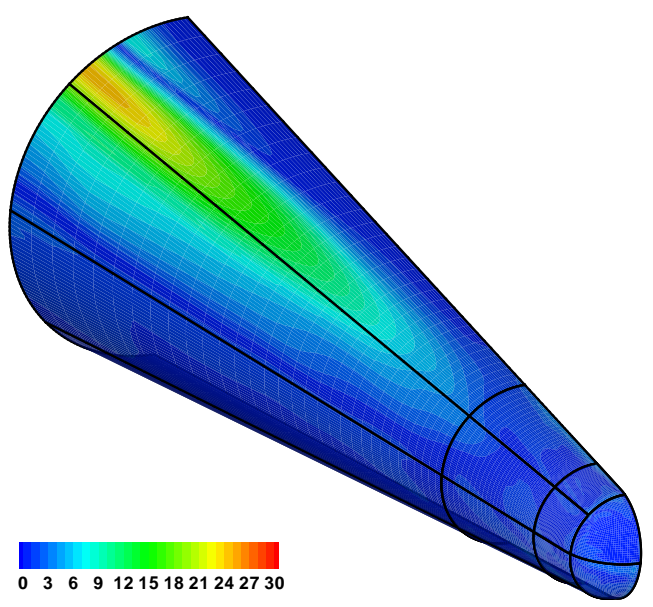

b) Moderately skewed.

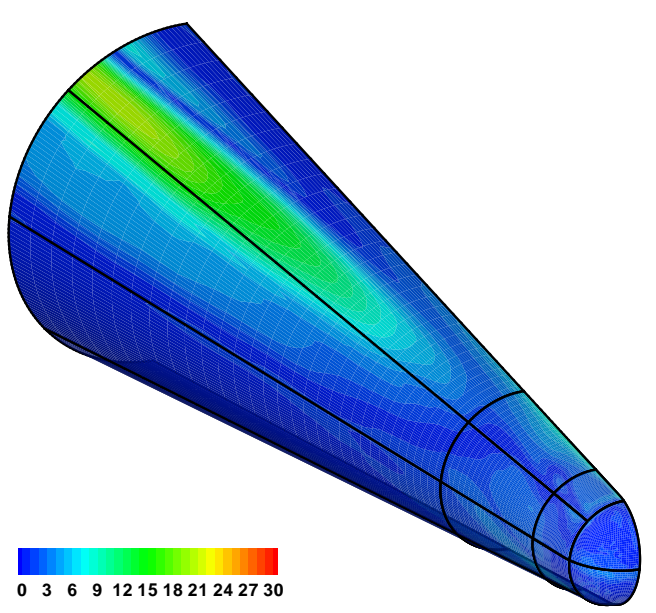

c) Highly skewed.

Fig. 19 Surface heating percentage differences between a quality mesh and poorly generated meshes.

the physics of the discretized volume. Thus, as expected, the differences between the quality and skewed meshes are large in a region where TLNS is predictably inaccurate.

The TLNS equations are typically used because the grid density required to accurately resolve cross-flow and 


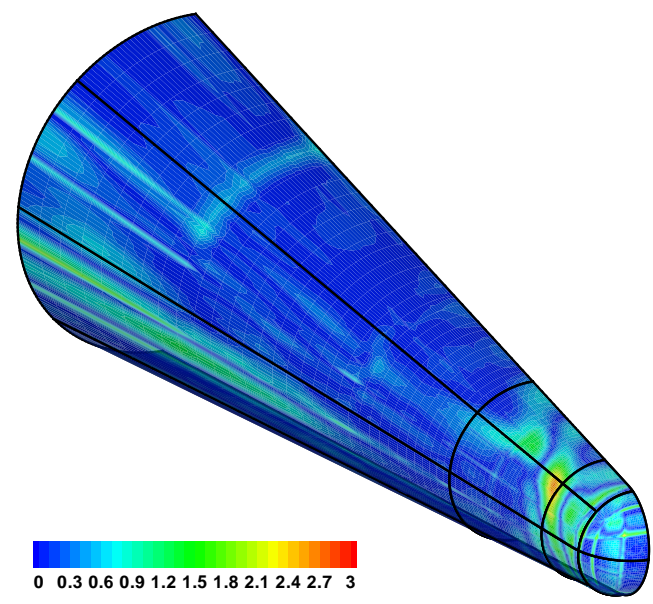

a) Stretched.

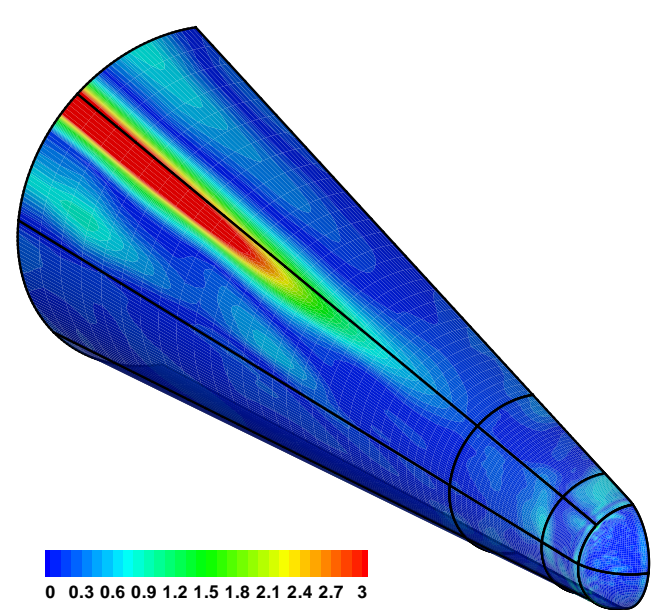

b) Moderately skewed.

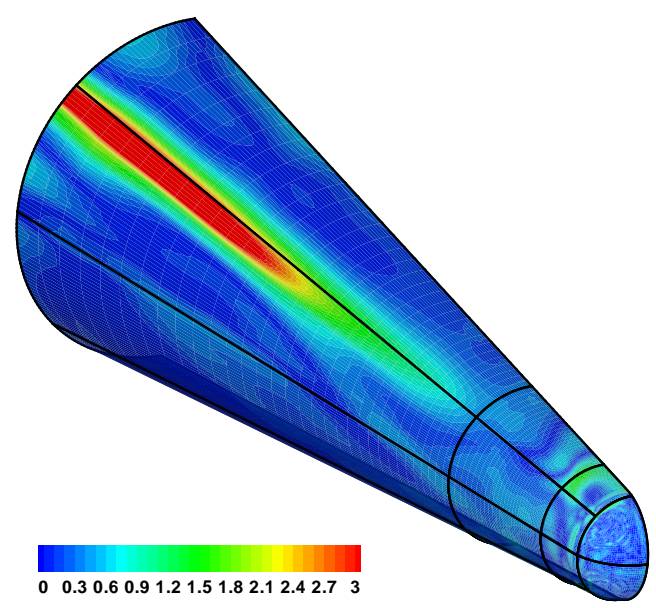

c) Highly skewed.

Fig. 20 Percent pressure comparisons between a quality mesh and poorly generated meshes.

streamwise viscous contributions to the boundary layer, is prohibitively large on modern computers. ${ }^{23,24}$ Each of the TLNS computations required approximately 25 hours to complete, on a DEC Alpha cluster. But the analysis indicates that TLNS may not be suitable for poorly constructed grids with LAURA.

A set of FNS simulations were performed using the same procedure as that of the TLNS. The FNS simulations required 32.5 hours each; a $33 \%$ increase in time to convergence because each iteration was approximately $33 \%$ longer in time. These FNS simulations required the same number of iterations, but an increase of $10 \%$ in memory. The results of the FNS surface heat transfer differences between the quality and poorly generated meshes are shown in figure 21, and tabulated maximums in table 3.

\begin{tabular}{ccc}
$\begin{array}{c}\text { Grids } \\
\text { Differenced from } \\
\text { Quality mesh }\end{array}$ & $\begin{array}{c}\text { Maximum } \\
\text { Pressure } \\
\text { (\% diff.) }\end{array}$ & $\begin{array}{c}\text { Maximum } \\
\text { Heating } \\
\text { (\% diff.) }\end{array}$ \\
\hline Stretched & 6.64 & 15.98 \\
Moderately skewed & 1.00 & 3.15 \\
Highly skewed & 1.05 & 3.87 \\
\hline
\end{tabular}

Table 3 FNS differences in heating and pressure between a quality mesh and poorly generated meshes.

With respect to the skewed meshes, aside from the blockto-block boundaries, the solutions are consistent with the FNS simulation on the quality grid. The largest difference is small, thus indicating that to obtain consistent simulations with skewed meshes to a solution produced on a quality mesh the full Navier-Stokes equations must be used. The skewed meshes also provide extreme heating differences as much as $50 \%$ along the block boundaries. Upon investigation of the LAURA software, the cross-derivative terms in the FNS equations use one sided differences at block boundaries. These skewed meshes were converged to machine zero, and no appreciable change occurred in the heating comparison. Thus, a rigid grid quality requirement has surfaced as a result of these computations; volume grids must be orthogonal to the wall at block boundaries for LAURA.

For the stretched mesh, an opposite trend from the TLNS simulation occurs. The heating differences are larger. Again, considering the pressure differences between the quality and stretched meshes as shown in figure 22, there are significant degradations in consistency of predicated pressure. These differences may be related to dispersions ${ }^{25,26}$ caused by the stretched mesh. Since LAURA does not have specific grid metrics to deal with stretched meshes, it appears that the cross-derivative terms are adversely affected by the grid stretching. Hence, neither the TLNS or FNS simulations appear to produce consistent results to the quality volume grid for grid stretching.

A final comparison of heating and pressure was performed between the TLNS and FNS simulations, and is shown in figure 23 and 24, respectively, and tabulated in table 4.

The heating and pressure differences for the high quality grid simulations has a maximum of $5 \%$ difference. Because these flow solutions have flow separation and reattachment, this $5 \%$ difference is considered acceptable, and any differences greater than $5 \%$ for the $3 \mathrm{D}$ simulations are evidence 


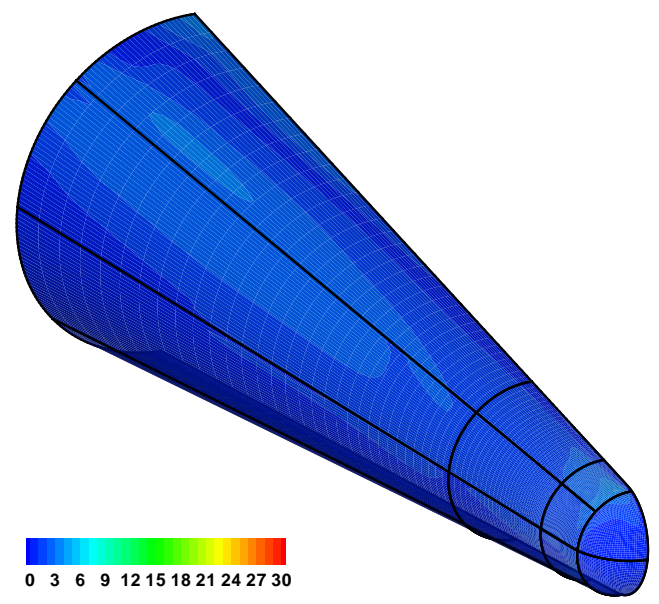

a) High quality.

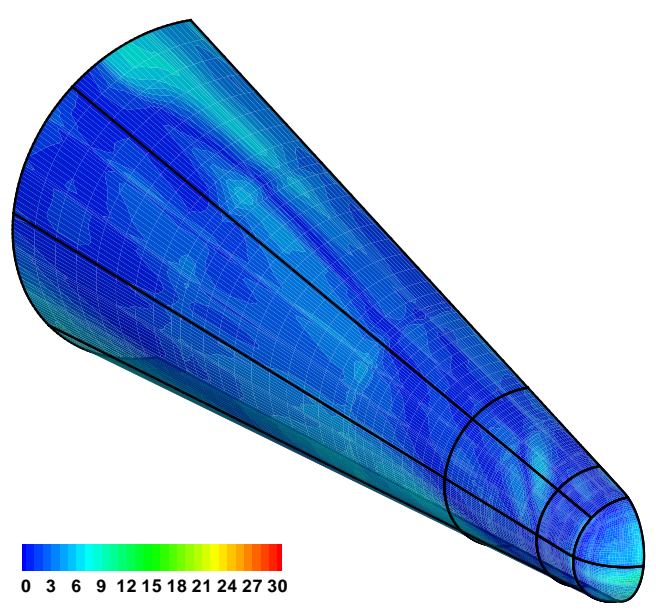

c) Stretched.

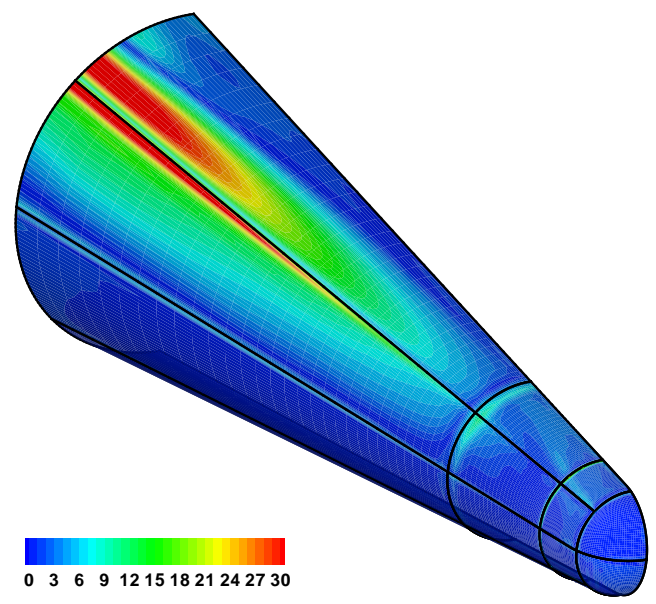

b) Moderately skewed.

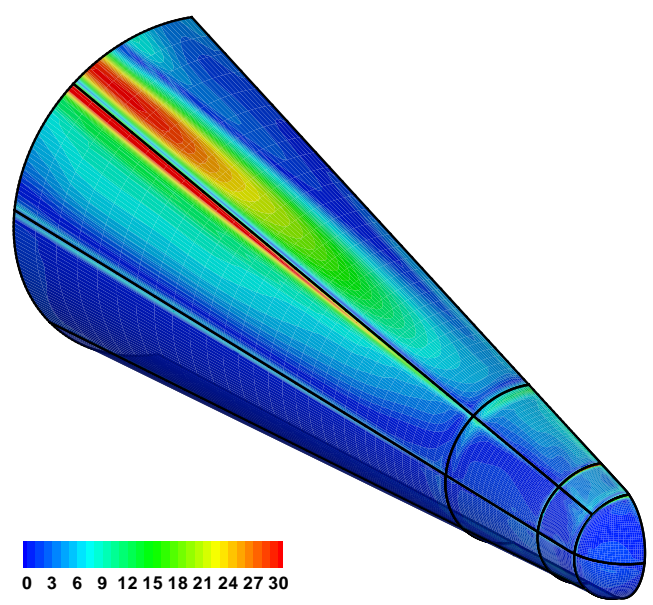

d) Highly skewed.

Fig. 23 Percentage surface heat transfer differences between FNS and TLNS simulations.

\begin{tabular}{ccc}
$\begin{array}{c}\text { Grids } \\
\text { Differenced } \\
\text { TLNS to FNS }\end{array}$ & $\begin{array}{c}\text { Maximum } \\
\text { Pressure } \\
\text { (\% error) }\end{array}$ & $\begin{array}{c}\text { Maximum } \\
\text { Heating } \\
\text { (\% error) }\end{array}$ \\
\hline Quality & 0.66 & 5.59 \\
Stretched & 6.35 & 14.9 \\
Moderately skewed & 16.1 & 35.4 \\
Highly skewed & 12.0 & 30.0 \\
\hline
\end{tabular}

Table 4 Differences in heating and pressure between FNS to TLNS simulations.

of grid metric degradation as it affects the flow solution. The poor quality meshes show significant differences in both surface heating and pressure. Based on the results presented thus far, the stretched mesh finds most applicability in the TLNS computations where cross-derivative terms are not used. The significant differences that are present for the FNS by comparison to the TLNS illustrates that these solutions are not consistent. Thus, stretched meshes could be used effectively in TLNS simulations, but the extent to stretching should be closely regulated and not permitted to exceed the current factor of 1.5 in the streamwise or crossflow directions.
The skewed meshes follow a different trend than the stretched grid. The TLNS simulations are significantly different than the quality mesh, but the FNS skewed mesh solutions are nearly identical to the quality mesh. If skewed meshes are used, flow simulations should employ FNS equations for accurate predictions. However, the matching block boundaries need to be orthogonal to the wall. Hence, skewed meshes near the wall are not valid flow discretizations for this sphere-cone geometry. Considering that this shape is simple, the data suggests that skewed meshes near the wall should be avoided. For if skewed meshes are used, the FNS simulations require more resources and time to obtain convergence. Practioners of CFD may consider the balancing of grid generation time to the work required to obtain the flow simulation. But for the cases presented here, the high quality grid required 3 hours to generate which represents $50 \%$ more time than generating the the low quality meshes. The TLNS simulations were completed with $33 \%$ less time and $10 \%$ less memory for a savings of 8 hours. Given the trade-off of generating a grid or computing a solution in this study, spending the time generating a grid is more economical. Hence, more efficient use of resources is obtained by utilizing high quality 


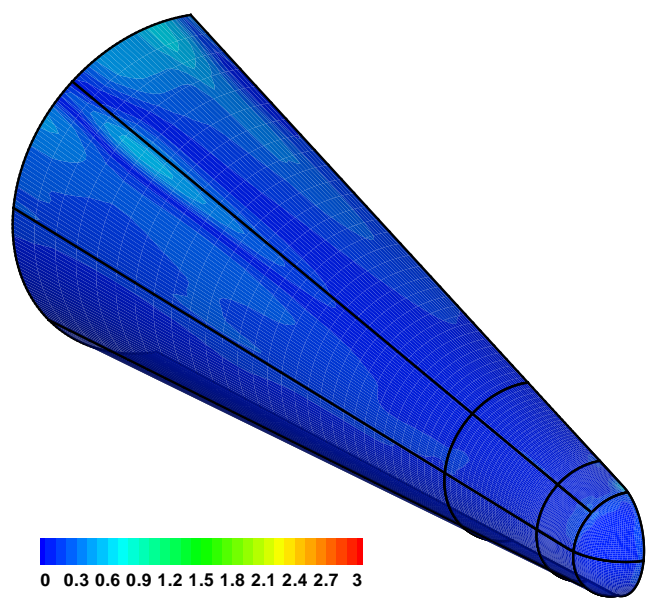

a) High quality.

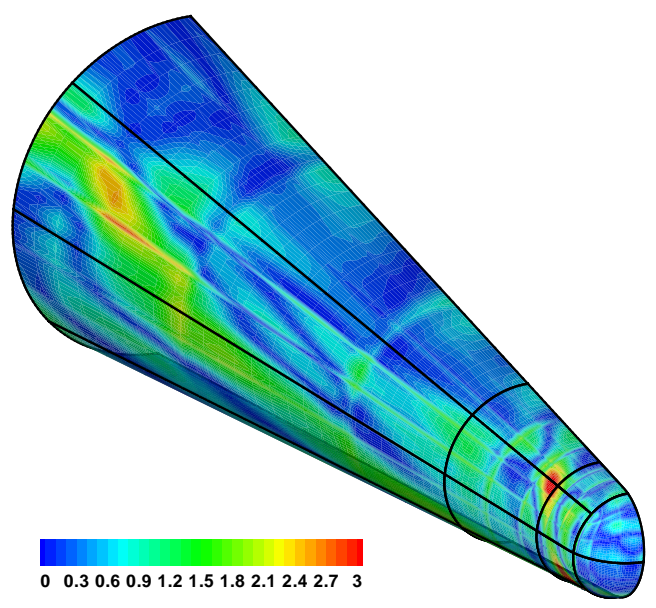

c) Stretched.

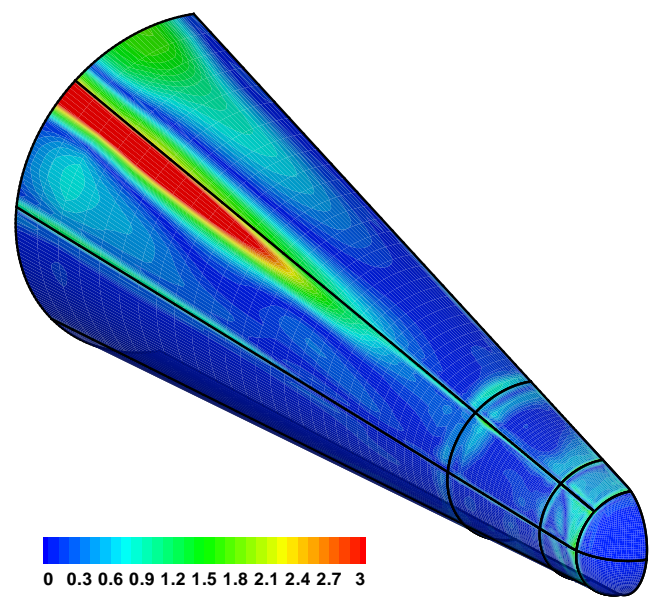

b) Moderately skewed.

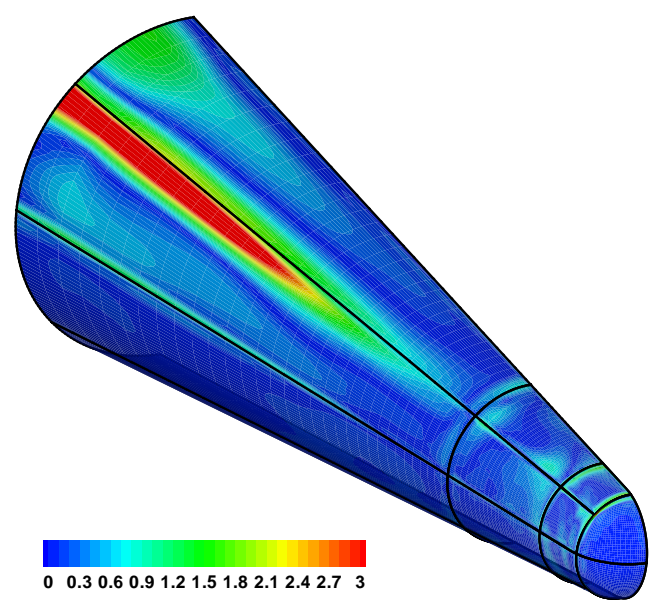

d) Highly skewed.

Fig. 24 Percentage surface pressure differences between FNS and TLNS simulations.

meshes and TLNS equations.

To assess grid convergence, Richardson Extrapolation was used to extrapolate two existing solutions of grid density to the next grid density. The grid density change between each that is used in this computation was scaled by a factor of 2 in each computational direction, for a total of a factor of eight. Prior to applying Richardson Extrapolation, the solutions are compared to one another at different grid densities. When comparing the high quality mesh surface solutions on the three grid densities, the difference between the coarse and medium peaked at $42.9 \%$ while the medium to fine resolution peaked at a $12.4 \%$ difference. Likewise, the remaining poor quality grids indicated some grid convergence as shown in table 5 .

Based on the convergence data, none of the solutions indicate grid convergence has been obtained. This is due to the difficulty of resolving leeside flow separation. By comparing an extrapolated solution from the medium grid to the fine grid solution, to the fine grid solution of each grid type, the solutions appear to be within $8 \%$ of fully converged, as shown in table 6 .

Considering the solutions are within the measurable differences between the high quality mesh and the remaining

\begin{tabular}{ccc}
$\begin{array}{c}\text { Volume } \\
\text { Grid } \\
\text { Type }\end{array}$ & $\begin{array}{c}\text { Coarse to } \\
\text { Medium } \\
\text { (\% error) }\end{array}$ & $\begin{array}{c}\text { Medium } \\
\text { to Fine } \\
\text { (\% error) }\end{array}$ \\
\hline High Quality & 42.9 & 12.4 \\
Stretched & 40.5 & 20.1 \\
Moderately skewed & 42.8 & 16.9 \\
Highly skewed & 44.8 & 16.6 \\
\hline
\end{tabular}

Table 5 Heating differences for coarse, medium, and fine grid densities using TLNS equations.

poor quality meshes, the comparisons of surface heating and pressure are valid. The TLNS computations with skewed grids have higher percentage heating differences with respect to the high quality mesh than the extrapolated grid convergence data for the skewed grids using the FNS computations. This indicates that if skewed grids are to be used for leeside flows, the FNS equations should be solved instead of the TLNS. Conversely, the FNS computations for the stretched grid shows differences in the heating that is greater than the extrapolated convergence data for the stretched grids, by comparison to the TLNS computations. Thus, improved consistency is possible using the 


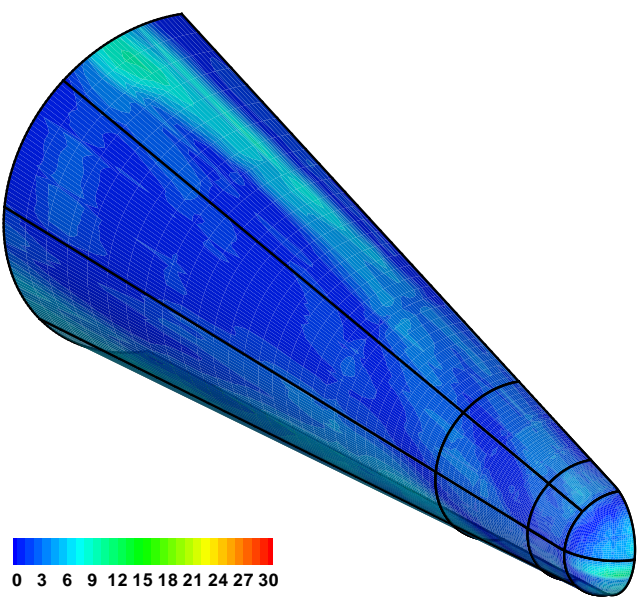

a) Stretched.

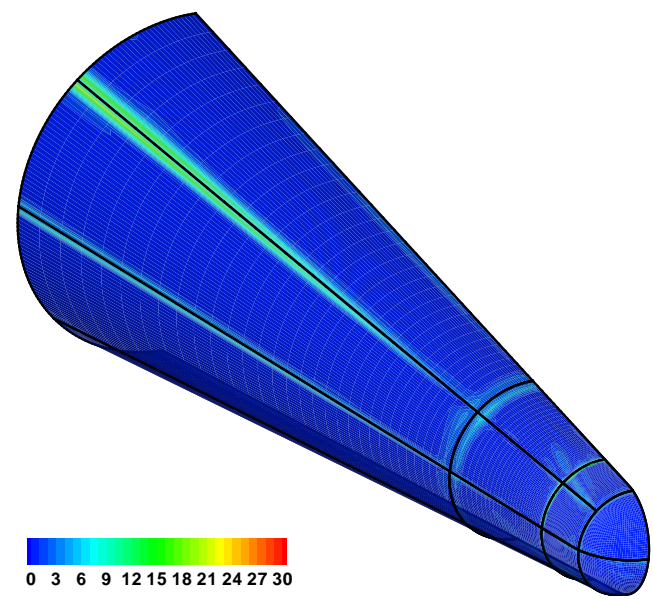

b) Moderately skewed.

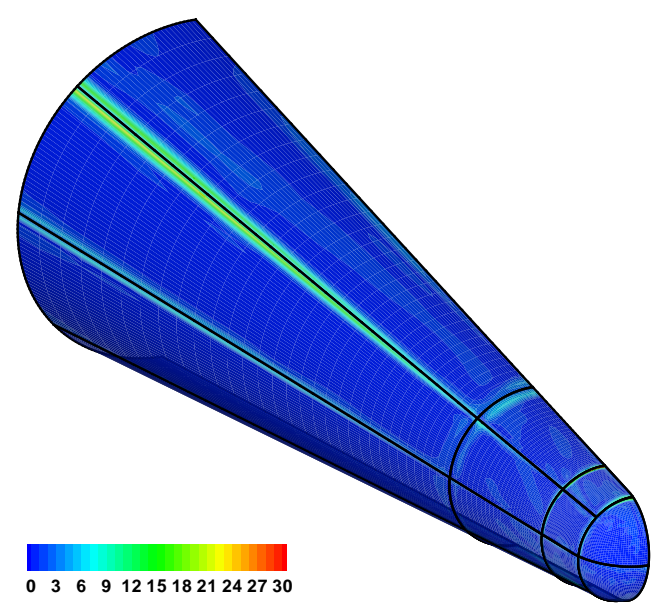

c) Highly skewed.

Fig. 21 FNS percent surface heating differences between a quality mesh and poorly generated meshes.

TLNS equations on stretched grids when using LAURA. However, with respect to the FNS and TLNS predicted heating differences for the high quality grid, the stretched and skewed meshes are significantly different from one another. In order to use the TLNS equations effectively as a

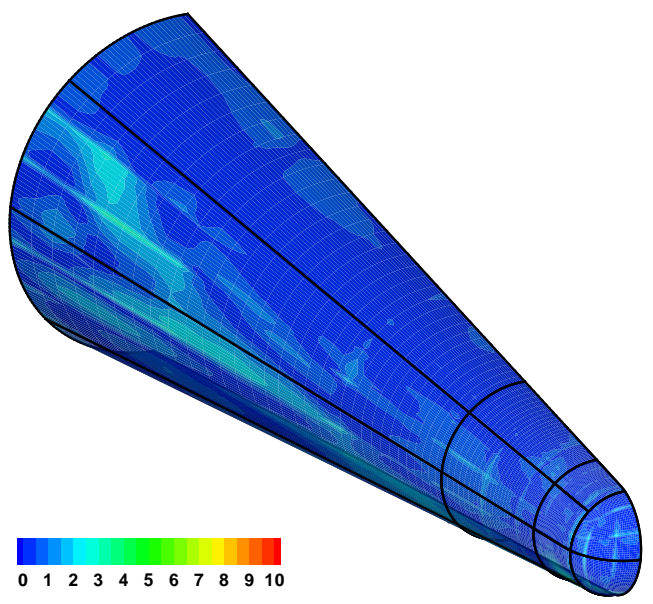

a) Stretched.

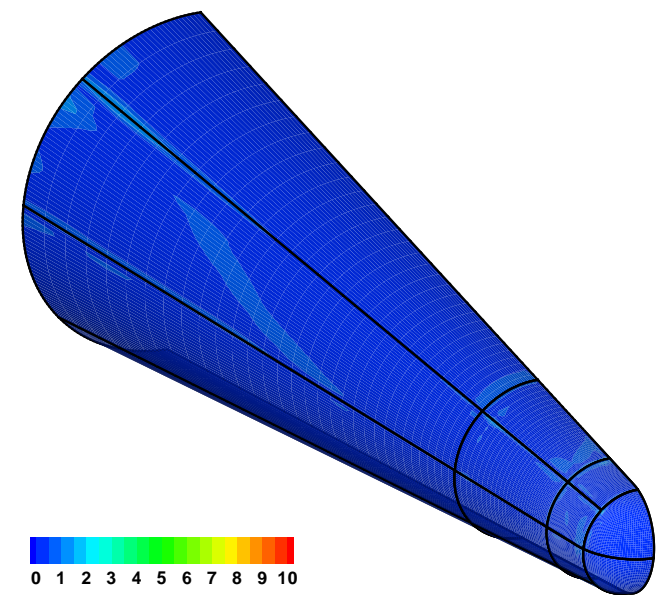

b) Moderately skewed.

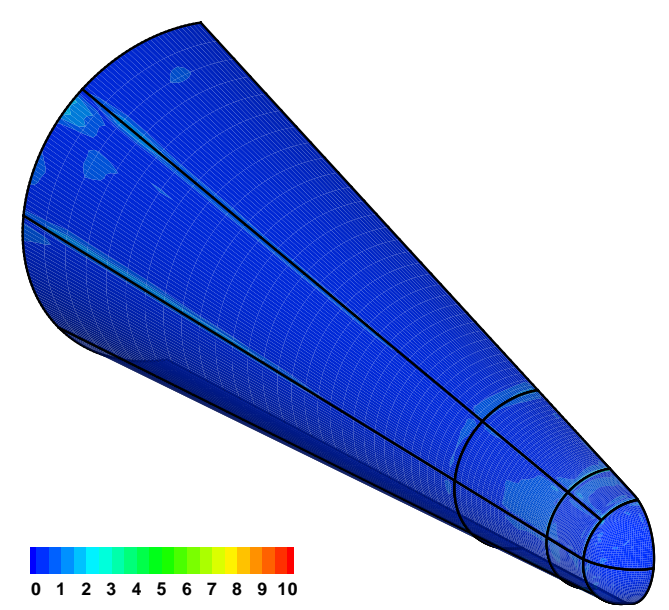

c) Highly skewed.

Fig. 22 FNS percent pressure differences between a quality mesh and poorly generated meshes.

reduction in complexity of the NS equations, there should be consistency between the FNS and TLNS computations. Only on the high quality mesh does this consistency exist. Thus, use of the high quality mesh as a baseline for comparisons is valid because a change in the equations that 


\begin{tabular}{ccc}
$\begin{array}{c}\text { Volume } \\
\text { Grid } \\
\text { Type }\end{array}$ & $\begin{array}{c}\text { TLNS - } \\
\text { Extrapolated } \\
\text { to Fine (\%) }\end{array}$ & $\begin{array}{c}\text { FNS - } \\
\text { Extrapolated } \\
\text { to Fine (\%) }\end{array}$ \\
\hline High Quality & 4.15 & 6.0 \\
Stretched & 6.70 & 7.95 \\
Moderately skewed & 5.63 & 5.95 \\
Highly skewed & 5.54 & 5.96 \\
\hline
\end{tabular}

Table 6 Grid convergence based on heating from extrapolating medium and fine solutions, and comparing to fine grid solution.

simulate the hypersonic flow field, does not reflect a significant percentage change in surface heat transfer.

\section{Grid Quality Correlation}

The primary goal of this work was to obtain information between a proposed grid quality metric and the ability of a grid to predict flow field and surface phenomenon. The GQ metric for each of the initial and solution adapted 3D volume grids is given in table 7 .

\begin{tabular}{ccc}
$\begin{array}{c}\text { Volume } \\
\text { Grid } \\
\text { Type }\end{array}$ & $\begin{array}{c}\text { GQ Before } \\
\text { Grid } \\
\text { Adaptation }\end{array}$ & $\begin{array}{c}\text { GQ After } \\
\text { Grid } \\
\text { Adaptation }\end{array}$ \\
\hline High Quality & 0.7271 & 0.7400 \\
Stretched & 0.6427 & 0.6536 \\
Moderately skewed & 0.6959 & 0.7070 \\
Highly skewed & 0.66008 & 0.6935 \\
\hline
\end{tabular}

Table 7 Grid Quality metric of the grids used in this study.

In order to determine a correlation, the surface heating and pressure differences from the high quality mesh by comparison to the low quality meshes are plotted with the grid quality metrics. Only the measure before grid adaptation will be considered for a correlation because the initial GQ metric is the consistent measure prior to any alterations of the volume grid. As shown in figure 25, if the skewness, or degree of orthogonality is considered, the differences between FNS and TLNS computations for the range of measured GQ show significant degradation of the solution. Conversely, the stretched mesh could be considered a good grid because of its proximity to the high quality mesh in the orthogonality correlation. But from the FNS computations, the stretched mesh has heating differences of approximately $15 \%$. Hence, orthogonality alone does not capture the overall quality of the grid.

Likewise, illustrated in figure 26, the stretching measure places the skewed meshes in the same range as the high quality mesh, but the stretched mesh is identified as not even close to the high quality mesh. Thus, stretching alone provides no conclusive correlation. Rather, both stretching and skewness measures have to be combined to isolate the high quality mesh from the poor quality meshes, so that some relationship between the effects of each quantity can result in a usable correlation. By dividing the skewness by the stretching, the combined effect is shown in figure 27, which still fails to correlate because the skewed meshes are

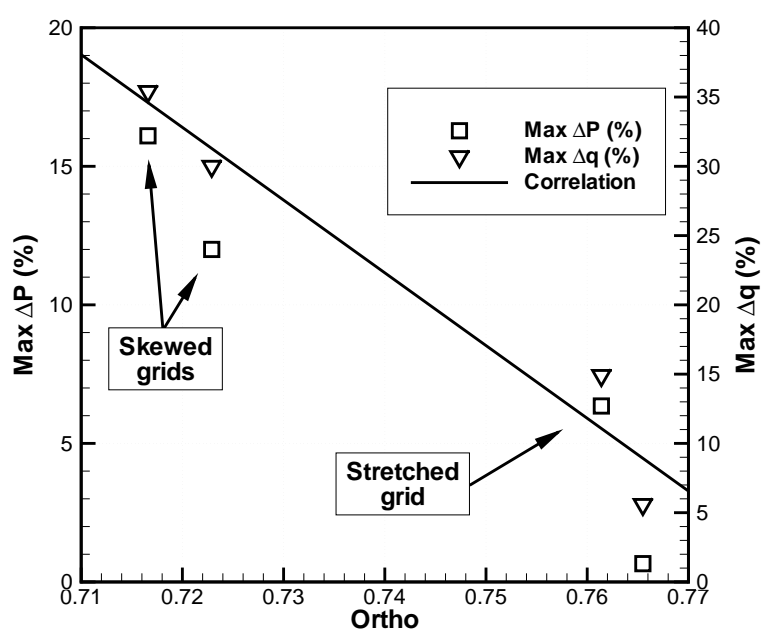

Fig. 25 Degree of orthogonality measure correlated to surface pressure and heat transfer simulation consistency between FNS and TLNS.

too close to one another.

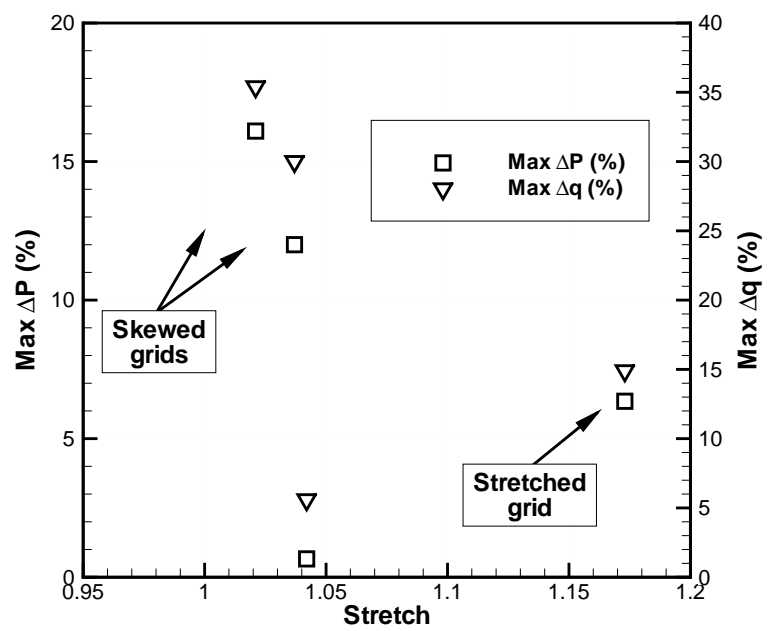

Fig. 26 Grid stretching measure correlated to surface pressure and heat transfer simulation consistency between FNS and TLNS.

However, simple inspection of the grids indicates that the highly skewed mesh is significantly different from the moderately skewed mesh. This difference is not identified by just stretching and skewness. To separate the effect of the skewness within the quality measures, an additional assessing value is incorporated into the overall GQ measure. This is the grid line straightness. The effect this has on the GQ metric is the separation of the highly skewed from the moderately skewed. The result of using the straightness factor is shown in figure 28. The problem with the proposed set of measures is that the solution differences do not correlate with the GQ. But the data does show a trend; the lower GQ values have reduced consistency of the sur- 


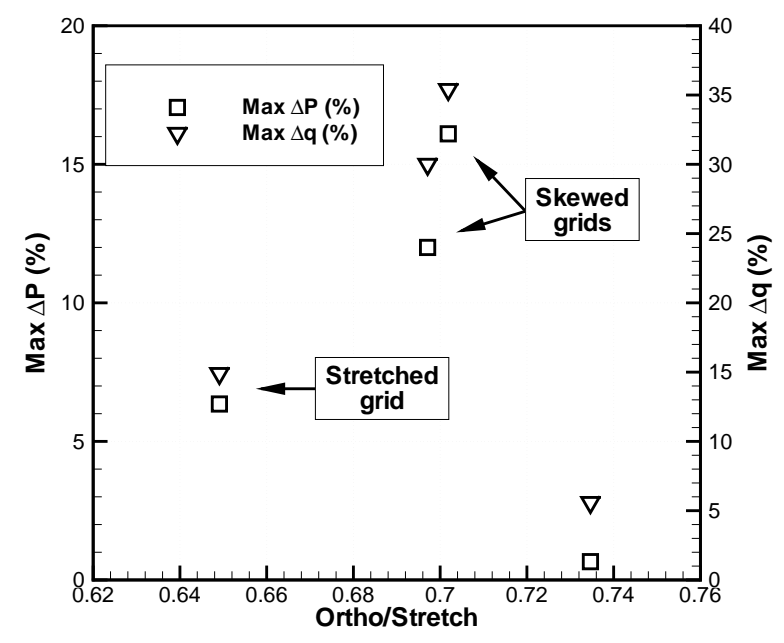

Fig. 27 Degree of orthogonality and stretching measures correlated to surface pressure and heat transfer simulation consistency between FNS and TLNS.

face heating and pressure. The GQ measure does illustrate that the three measures can be used in the combination because higher stretching and increased skewness does reflect low GQ values. The choice of global quantities may be the source of the non-correlative nature of the data obtained. Therefore, further refinement of the individual measures is warranted. For this study, using LAURA applied to a slender spherically blunted cone in 3D with flow separation and attachment, the region of acceptable GQ measures of grids that could be used to accurately predict flow field data is above the 0.72 value. This relates to heating accuracy within $5 \%$ and pressure accuracy within $2 \%$, as shown by the red line in figure 28. Though the GQ measure of 0,72 is not a threshold, reduced GQ measure can result in the degradation of flow field prediction accuracy as indicated by the data.

\section{Concluding Remarks}

A method for determining the quality of a structured grid with respect to global measures is presented, consisting of the product of skewness and straightness divided by the stretching. The grid quality metric, which ranges from 1 at the best to 0 at the worst with typical grids falling in the range [0.6-0.8], is used to characterize structured grids for the simulation of hypersonic flow. A sphere-cone at wind tunnel conditions was used to evaluate the applicability of the grid quality measure.

The grids used in this study primarily vary the skewness and stretching, although straightness is impacted to a lesser degree, to determine the effect of grid quality on computed surface heat transfer and pressure predictions. Comparisons of heat transfer rate predicted via grid converged solutions indicate that domain discretizations with the least skewness, least stretching, and straightest grid lines result in heating predictions that are consistent with the least computational resources required. Thin-layer Navier-Stokes

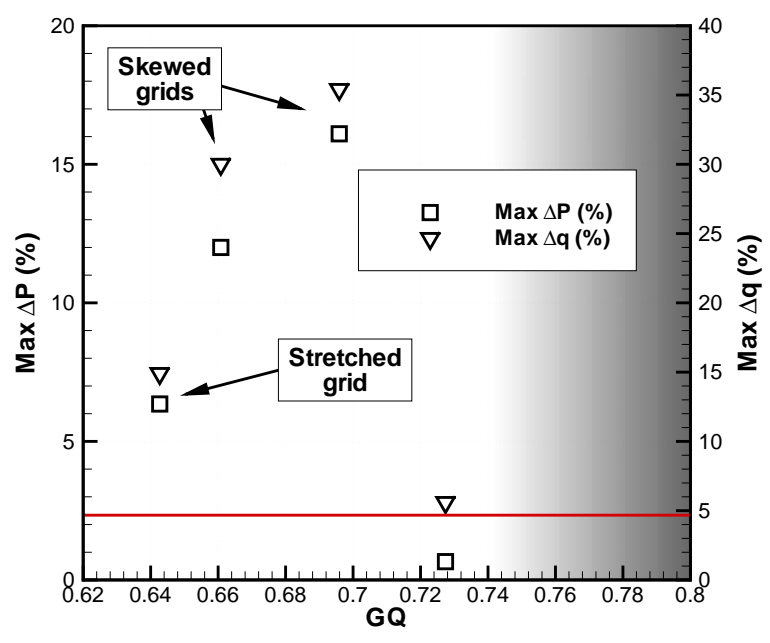

Fig. 28 GQ measure correlated to surface pressure and heat transfer simulation consistency between FNS and TLNS.

(TLNS) equations are usable on high quality meshes as compared to the need to use full Navier-Stokes (FNS) equations for some low quality meshes. A grid that exhibits high quality features also has the largest grid quality metric. As the skewness of the grid is increased, the accuracy of the heating prediction decreased. The grid with stretching does converge, but does not accurately predict pressure and surface heat transfer when using the FNS equations.

Structured grids of reasonable quality can be used to obtain accurate aerothermodynamic simulations, but as the grid quality increases the types of equations in use can be simplified. The leeside separation on the 3D spherecone is the most difficult feature of the flow field to predict as compared to the windside. If solutions for the leeside can be shown to be consistent when using the TLNS equations, then the use of TLNS in a preliminary design process could provide detailed analyses in a timely manner. The higher quality grid tends to reduce the magnitude of crossderivative grid metrics. Thus, using FNS or TLNS produces similar results for the high quality grid. However, the skewed meshes required the FNS to obtain accurate simulations. Under the conditions of the sphere-cones analyzed in this paper, the FNS requires $33 \%$ more time to converge for the same number of iterations, and approximately $10 \%$ more computer memory than TLNS. Hence, maximizing the use of TLNS may enable the incorporation of computational fluid dynamics in a preliminary design process.

The grid quality measure does provide a mechanism to combine the effects of multiple grid quality measures that may lead to a usable grid quality measure, even though it is not the most definitive at assessing the capacity of a grid to produce accurate flow solutions. Only through use can the measure be refined to provide enhanced correlation of grid quality to flow properties. 


\section{Acknowledgement}

The author wishes to express his sincere appreciation to Dr. William A. Wood and Dr. Peter A. Gnoffo of the Aerothermodynamics Branch of NASA Langley Research Center for their editorial comments during the preparation of this paper.

\section{References}

${ }^{1}$ Gnoffo, P. A., Weilmuenster, K. J., Hamilton II, H. H., Olynick, D. R., and Venkatapathy, E., "Computational Aerothermodynamic Design Issues for Hypersonic Vehicles," AIAA Paper 97-2473, June 1997.

${ }^{2}$ Kleb, W. L. and Weilmuenster, K. J., "Characteristics of the Shuttle Orbiter Leeside Flow During a Reentry Condition,” AIAA Paper 92-2951, July 1992.

${ }^{3}$ Weilmuenster, K. J., Gnoffo, P. A., and Greene, F. A., "NavierStokes Simulations of Orbiter Aerodynamic Characteristics Including Pitch Trim and Bodyflap," AIAA Journal of Spacecraft and Rockets, Vol. 31, No. 3, May-June 1994, pp. 355-366.

${ }^{4}$ Bonner, E., Clever, W., and Dunn, K., "Aerodynamic Preliminary Analysis System II. Part I - Theory,” NASA-CR 165627, 1989.

${ }^{5}$ Divan, P. and Sova, G., "Aerodynamic Preliminary Analysis System II. Part II - Users Manual,” NASA Contractor Report 165628, 1989.

${ }^{6}$ Taylor, S., "MARK IV Supersonic-Hypersonic Arbitrary-Body Program Modifications and Computer Graphics V. I: Surface Streamlin Tracing," AFWAL TR-80-3117, August 1980.

${ }^{7}$ Edington, L. and Williams, R. M., "PAYCOS, A Multidisciplinary Sizing Code for Hypersonic Vehicles," AIAA Paper 92-4564, August 1992.

${ }^{8}$ Thompson, J. F., Warsi, Z. U. A., and Mastin, C. W., Numerical Grid Generation Foundations and Applications, North-Holland, 1st ed., 1985, pp. 171-184.

${ }^{9}$ Strang, W. Z., "QBERT: A Grid Evaluation Code," AFWAL Technical Memorandum 99-193, Wright Research and Development Center Report, July 1988.

${ }^{10}$ Olynick, D. D. R., "Importance of 3-D Grid Resolution and Structure for Calculating Reentry Heating Environments," AIAA Paper 961857, June 1996.

${ }^{11}$ Gnoffo, P. A., "An Upwind-Biased Point-Implicit Relaxation Algorithm for Viscous, Compressible Perfect-Gas Flows," NASA Technical Paper 2953, February 1990.

${ }^{12}$ Parmley, K. L., Dannenhoffer III, J. F., and Weatherill, N. P., "Techniques for the Visualization of Computational Grids," AIAA Paper 933353-CP, July 1993.

${ }^{13}$ Gordon, W. J. and Hall, C. A., "Construction of Curvilinear Coordinate Systems and Applications to Mesh Generation," International Journal of Numerical Methods in Engineering, Vol. 3, No. 7, July 1973, pp. 461-477.

${ }^{14}$ Alter, S. J., The Volume Grid Manipulator (VGM): A Grid Reusability Tool, No. 4772, April 1997.

${ }^{15}$ Alter, S. J., The Volume Grid Manipulator (VGM): A Grid Reusability Tool, chap. 9, No. 4772, NASA Contractor Report, April 1997, pp. 61-75.

${ }^{16}$ Sorenson, R. L. and Alter, S. J., "3DGRAPE/AL: The Ames/Langley Technology Upgrade," Surface Modeling, Grid Generation, and Related Issues in Computational Fluid Dynamic (CFD) Solutions, edited by Y. K. Choo, No. CP-3291, NASA, May 1995, pp. 447-462.

${ }^{17}$ Merski, N. R., "Global Aeroheating Wind-Tunnel Measurements Using Improved Two-Color Phosphor Thermography Method,' Journal of Spacecraft and Rockets, Vol. 36, No. 2, March-April 1999, pp. 160170.

${ }^{18}$ Roe, P. L., "Approximate Riemann Solvers, Parameter Vectors, and Difference Schemes," Journal of Computational Physics, Vol. 43, No. 10, October 1981, pp. 357-372.

${ }^{19}$ Harten, A., "High Resolution Schemes for Hyperbolic Conservation Laws," Journal of Computational Physics, Vol. 49, No. 3, March 1983, pp. 357-393.
${ }^{20}$ Yee, H. C., "On Symmetric and Upwind TVD Schemes," NASA Technical Memorandum 86842, September 1985.

${ }^{21}$ Fay, J. A. and Riddell, F. R., "Theory of Stagnation Point Heat Transfer in Dissociated Air," Journal of the Aeronautical Sciences, Vol. 25, No. 2, February 1958, pp. 73-85.

${ }^{22}$ Roache, P. J., Computational Fluid Dynamics, Hermosa Publishers, 1976, pp. 177-178.

${ }^{23}$ Baldwin, B. S. and Lomax, H., "Thin Layer Approximation and Algebraic Model for Separated Turbulent Flows," AIAA Paper 78-0257, 1978.

${ }^{24}$ Anderson, D. A., Tannehill, J. C., and Pletcher, R. H., Сomputational Fluid Dynamics and Heat Transfer, chap. 8, Taylor\& Francis, Washington, D. C., 1st ed., 1984, pp. 421-422.

${ }^{25}$ Roache, P. J., Computational Fluid Dynamics, Hermosa Publishers, 1976, p. 45.

${ }^{26}$ Hoffmann, K. A. and Chiang, S. T., Computational Fluid Dynamics for Engineers, Vol. 1, Engineering Education System ${ }^{T M}$, Austin, Texas, 1st ed., 1989, pp. 143-145. 